\title{
Equalization With Oversampling in Multiuser CDMA Systems
}

\author{
Bojan Vrcelj, Member, IEEE, and P. P. Vaidyanathan, Fellow, IEEE
}

\begin{abstract}
Some of the major challenges in the design of newgeneration wireless mobile systems are the suppression of multiuser interference (MUI) and inter-symbol interference (ISI) within a single user created by the multipath propagation. Both of these problems were addressed successfully in a recent design of A Mutually Orthogonal Usercode-Receiver (AMOUR) for asynchronous or quasisynchronous code division multiple access (CDMA) systems. AMOUR converts a multiuser CDMA system into parallel single-user systems regardless of the multipath and guarantees ISI mitigation, irrespective of the channel null locations. However, the noise amplification at the receiver can be significant in some multipath channels. In this paper, we propose to oversample the received signal as a way of improving the performance of AMOUR systems. We design Fractionally Spaced AMOUR (FSAMOUR) receivers with integral and rational amounts of oversampling and compare their performance with the conventional method. An important point that is often overlooked in the design of zero-forcing channel equalizers is that sometimes, they are not unique. This becomes especially significant in multiuser applications where, as we will show, the nonuniqueness is practically guaranteed. We exploit this flexibility in the design of AMOUR and FSAMOUR receivers and achieve noticeable improvements in performance.
\end{abstract}

Index Terms-Code division multiaccess, fractionally spaced equalizers, MIMO systems, multiuser channels.

\section{INTRODUCTION}

$\mathbf{T}$ HE performance of the new-generation wireless mobile systems is limited by the multiuser interference (MUI) and inter-symbol interference (ISI) effects. The interference from other users (MUI) has traditionally been combated by the use of orthogonal spreading codes at the transmitter [16]; however, this orthogonality is often destroyed after the transmitted signals have passed through the multipath channels. Furthermore, in the multichannel uplink scenario, exact multiuser equalization is possible only under certain conditions on the channel matrices [13]. The alternative approach is to suppress MUI statistically; however, this is often less desirable.

A recent major contribution in this area is the development of A Mutually Orthogonal Usercode-Receiver (AMOUR) by Giannakis et al. [4], [22]. Their approach aims at eliminating MUI

Manuscript received May 2, 2003; revised May 12, 2004. This work was supported in part by the United States Office of Naval Research under Grant N00014-99-1-1002. The associate editor coordinating the review of this manuscript and approving it for publication was Dr. Joseph Tabrikian.

B. Vrcelj was with the Department of Electrical Engineering, California Institute of Technology, Pasadena, CA 91125 USA. He is now with Qualcomm, Inc., San Diego, CA 92121 USA (e-mail: bojan@qualcomm.com).

P. P. Vaidyanathan is with the Department of Electrical Engineering, California Institute of Technology, Pasadena, CA 91125 USA (e-mail: ppvnath@ systems.caltech.edu).

Digital Object Identifier 10.1109/TSP.2005.845494 deterministically and, at the same time, mitigating the undesired effects of multipath propagation for each user separately. The former is achieved by carefully designing the spreading codes at the transmitters and the corresponding equalization structures at the receivers. In [3] and [4], AMOUR systems were designed for multiuser scenarios with uniform information rates, whereas in [22], the idea was extended for the case when different users communicate at different rates. One clear advantage of this over the previously known methods is that MUI elimination is achieved irrespective of the channel nulls. Moreover, ISI cancellation can be achieved using one of the previously known methods for blind channel equalization [4]. In summary, AMOUR can be used for deterministic MUI elimination and fading mitigation, regardless of the (possibly unknown) multipath uplink channels.

In this work, we consider a possible improvement of the basic AMOUR-CDMA system described in [3]. The proposed structure consists of a multiple-transmitter, multiple-receiver AMOUR system with signal oversampling at the receivers. This equalizer structure can be considered to be a fractionally spaced equalizer (FSE) [12] and, thus, the name Fractionally Spaced AMOUR (FSAMOUR). We consider two separate cases: integral and rational oversampling ratios. Even though integral oversampling can be viewed as a special case of rational oversampling, we treat them separately since the analysis of the former is much easier. In particular, when the amount of oversampling is a rational number, we need to impose some additional constraints on the systems parameters in order for the desirable channel-invariance properties of conventional AMOUR systems to carry through. In contrast, no additional constraints are necessary in the integral case.

An additional improvement of multiuser communication systems is achieved by exploiting the fact that zero-forcing channel equalizers are not unique, even for fixed equalizer orders. This nonuniqueness allows us to choose such zero forcing equalizers (ZFEs) that will reduce the noise power at the receiver. Note that this improvement technique is available in both AMOUR and FSAMOUR systems. As in other areas where FSEs find their application [12], [15], [17], the advantages over the conventional symbol-spaced equalizers (SSE) are lower sensitivity to the synchronization issues and freedom in the design of ZFEs. We will see that the aforementioned additional freedom translates to better performance of FSAMOUR ZFEs.

In Section II, we provide an overview of the AMOUR-CDMA systems, as introduced by Giannakis and others. Our approach to the system derivation provides an alternative point of view and leads to notable simplifications, which prove essential in the derivation of FSEs. In Section III, we design the FSAMOUR 
system with an integral amount of oversampling. The system retains all the desired properties of conventional AMOUR and provides additional freedom in the design of ZF solutions, which corresponds to finding left inverses of tall matrices with excess rows. This freedom is further exploited, and the corresponding improvement in performance over the AMOUR system is reported in the subsection with the experimental results. In Section IV, we generalize the notion of FSAMOUR to the case of fractional oversampling at the receiver. If the amount of oversampling is given by $(M+1) / M$ for a large integer $M$, the computational overhead in terms of the increased data rate at the receiver becomes negligible. Experimental results in Section IV-E confirm that the improvements in the equalizer performance can be significant, even if the oversampling is by $6 / 5$.

\section{A. Notations}

If not stated otherwise, all notations are as in [14]. We use boldface letters to denote matrices. Superscripts $(\cdot)^{T}$ and $(\cdot)^{\dagger}$, respectively, denote the transpose and the transpose-conjugate operations on matrices. The identity matrix of size $N \times N$ is denoted by $\mathbf{I}_{N}$. Let $r(z)$ be the rank of a polynomial matrix in $z$. The normal rank is defined as the maximum value of $r(z)$ in the entire $z$ plane.

In a block diagram, the $M$-fold decimation and expansion operations will be denoted by encircled symbols $\downarrow M$ and $\uparrow M$, respectively.

The polyphase decomposition [14] plays a significant role in the following. If $F(z)$ is a transfer function, then it can be written in the Type-1 polyphase form as

$$
F(z)=\sum_{k=0}^{M-1} z^{-k} F_{k}\left(z^{M}\right)
$$

where $F_{k}(z)$ is the $k$ th Type- 1 polyphase component of $F(z)$. A similar expression defines the Type-2 polyphase components, namely, $G(z)=\sum_{k=0}^{M-1} z^{k} G_{k}\left(z^{M}\right)$.

\section{AMOUR CDMA SYSTEMS}

The structure in Fig. 1 describes the AMOUR-CDMA system for $M$ users, i.e., $M$ transmitters and $M$ potential receivers. The upper part of the figure shows the $m$ th transmitter followed by the uplink channel corresponding to the $m$ th user, and the lower part shows the receiver tuned to the user $m$. The symbol stream $s_{m}(n)$ is first blocked into a vector signal $\mathbf{s}_{m}(n)$ of length $K$. This signal is upsampled by $P>K$ and passed through a synthesis filterbank of spreading codes $\left\{C_{m, k}(z)\right\}_{k=0}^{K-1}$; thus, each of the transmitters introduces redundancy in the amount of $P / K$. It is intuitively clear that this redundancy serves to facilitate the user separation and channel equalization at the receiver. While larger $K$ serves to reduce the bandwidth expansion $P / K$, for any fixed $K$, there is the minimum required $P$ (a function of $K$ and the channel order $L$ ) for which user separation and perfect channel equalization is possible. It will become clear that for large values of $K$, the overall bandwidth expansion tends to $M$, i.e., its minimum value in a system with $M$ users. It is shown in [22] that a more general system where different users communicate at different information rates can be reduced to the single rate system. Therefore, in the following, we consider the case where $K$ and $P$ are fixed across different users.

The channels $H_{m}(z)$ are considered to be finite impulse response (FIR) of order $\leq L$. The $m$ th receiver is functionally divided into three parts: filterbank $\left\{G_{m, j}(z)\right\}_{j=0}^{J-1}$ for MUI cancellation, block $\mathbf{V}_{m}^{-1}$, which is supposed to eliminate the effects of $\left\{C_{m, k}(z)\right\}$ and $\left\{G_{m, j}(z)\right\}$ on the desired signal $s_{m}(n)$, and the equalizer $\boldsymbol{\Gamma}_{m}$ aimed at reducing the ISI introduced by the multipath channel $H_{m}(z)$. Filters $G_{m, j}(z)$ are chosen to be FIR and are designed jointly with $\left\{C_{m, k}(z)\right\}$ to filter out the signals from the undesired users $\mu \neq m$. The choice of $\left\{C_{m, k}(z)\right\}$ and $\left\{G_{m, j}(z)\right\}$ is completely independent of the channels $H_{m}(z)$ and depends only on the maximum channel order $L$. Therefore, in this paper, we assume that CSI is available only at the block equalizers $\boldsymbol{\Gamma}_{m}$. If the channels are altogether unknown, some of the well-known blind equalization techniques [1], [2], [8], [10] can be readily incorporated at the receiver (see [4] and [9]). While the multiuser system described here is ultimately equivalent to the one in [3], the authors believe that this design provides a new way of looking at the problem. Furthermore, the simplifications introduced by the block notation will prove instrumental in Sections III and IV.

In the following, we design each of the transmitter and receiver building blocks by rewriting them in a matrix form. The banks of filters $\left\{C_{m, k}(z)\right\}$ and $\left\{G_{m, j}(z)\right\}$ can be represented in terms of the corresponding $P \times K$ and $J \times P$ polyphase matrices $\hat{\mathbf{C}}_{m}$ and $\mathbf{G}_{m}$, respectively, [14]. The $(j, i)$ th element of $\mathbf{G}_{m}$ is given by $g_{m, j}(i)$ and the $(i, k)$ th element of $\hat{\mathbf{C}}_{m}$ by $c_{m, k}(i)$. Note that the polyphase matrices $\hat{\mathbf{C}}_{m}$ and $\mathbf{G}_{m}$ become constant once we restrict the filters $C_{m, k}(z)$ and $G_{m, j}(z)$ to length $P$.

The system from Fig. 1 can now be redrawn as in Fig. 2(a), where the receiver block is defined as $\mathcal{T}_{m} \triangleq \Gamma_{m} \mathbf{V}_{m}^{-1} \mathbf{G}_{m}$. The $P \times P$ block in Fig. 2(a), consisting of the signal unblocking, filtering through the $m$ th channel, and blocking, can be equivalently described as in Fig. 2(b). Namely, it can be shown [14] that the corresponding $P \times P$ LTI system is given by the following matrix:

$$
\hat{\mathbf{H}}_{m}=\left[\mathbf{H}_{m} \mathbf{X}(z)\right] .
$$

Here, we denote by $\mathbf{H}_{m}$ the $P \times P-L$ full-banded lower triangular Toeplitz matrix

$$
\mathbf{H}_{m}=\left[\begin{array}{cccc}
h_{m}(0) & 0 & \cdots & 0 \\
\vdots & h_{m}(0) & & \vdots \\
h_{m}(L) & \vdots & \ddots & 0 \\
0 & h_{m}(L) & & 0 \\
\vdots & \vdots & \ddots & \vdots \\
0 & 0 & \cdots & h_{m}(L)
\end{array}\right]
$$

and $\mathbf{X}(z)$ is the $P \times L$ block that introduces the IBI. By choosing the last $L$ samples of the spreading codes $\left\{C_{m, k}(z)\right\}$ to be zero, $\hat{\mathbf{C}}_{m}$ is of the form $\hat{\mathbf{C}}_{m}=\left[\mathbf{C}_{m}^{T} \mathbf{0}^{T}\right]^{T}$ with the $L \times K$ zero- 

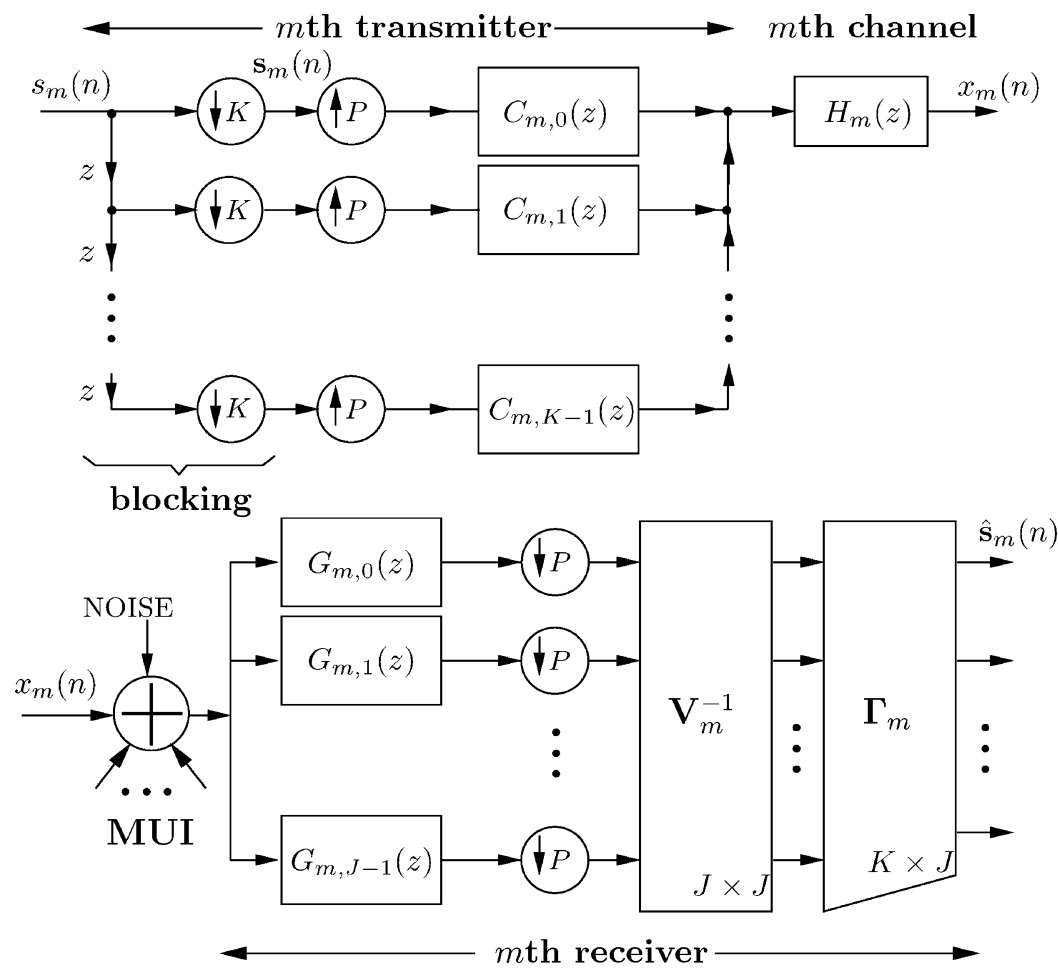

Fig. 1. Discrete-time equivalent of a baseband AMOUR system.

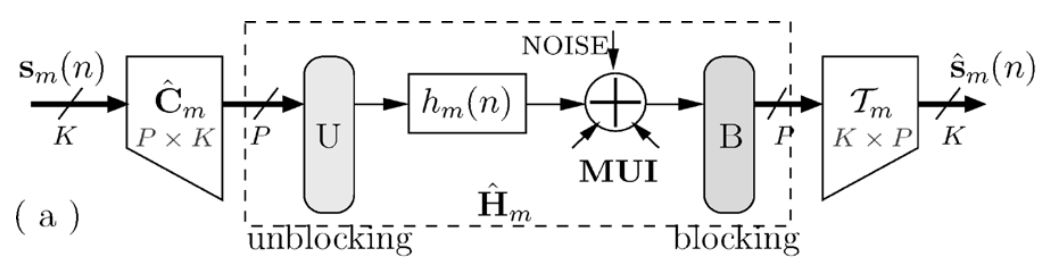

( b )
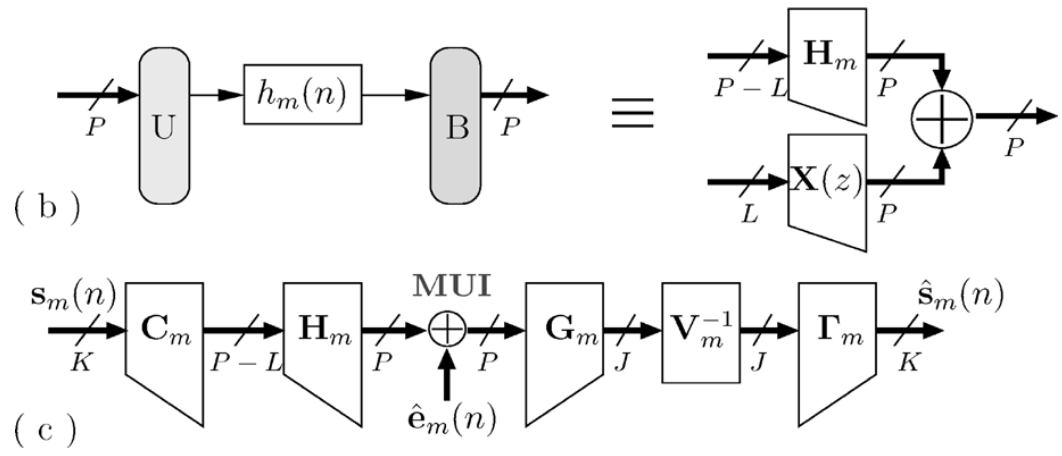

Fig. 2. Equivalent drawings of a symbol-spaced AMOUR system.

block positioned appropriately to eliminate the IBI block $\mathbf{X}(z)$. Namely, we have

$$
\hat{\mathbf{H}}_{m} \hat{\mathbf{C}}_{m}=\left[\begin{array}{ll}
\mathbf{H}_{m} & \mathbf{X}(z)
\end{array}\right] \cdot\left[\begin{array}{c}
\mathbf{C}_{m} \\
\mathbf{0}
\end{array}\right]=\mathbf{H}_{m} \mathbf{C}_{m}
$$

Therefore, the IBI-free equivalent scheme is shown in Fig. 2(c), with the noise vector signal $\hat{\mathbf{e}}_{m}(n)$ obtained by blocking the noise from Fig. 2(a). Next, we use the fact that full-banded Toeplitz matrices can be diagonalized by Vandermonde matrices. Namely, let us choose

$$
\mathbf{G}_{m}=\left[\begin{array}{cccc}
1 & \rho_{m, 0}^{-1} & \cdots & \rho_{m, 0}^{-P+1} \\
1 & \rho_{m, 1}^{-1} & \cdots & \rho_{m, 1}^{-P+1} \\
\vdots & \vdots & & \vdots \\
1 & \rho_{m, J-1}^{-1} & \cdots & \rho_{m, J-1}^{-P+1}
\end{array}\right], \quad \text { for } \rho_{m, j} \in \mathbb{C}
$$


denote by $\boldsymbol{\Theta}_{m}$ the first $P-L$ columns of $\mathbf{G}_{m}$, and define the diagonal matrix

$$
\mathcal{H}_{m}\left(\boldsymbol{\rho}_{m}\right) \triangleq \operatorname{diag}\left[H_{m}\left(\rho_{m, 0}\right), H_{m}\left(\rho_{m, 1}\right), \cdots, H_{m}\left(\rho_{m, J-1}\right)\right]
$$

with the argument defined as $\boldsymbol{\rho}_{m} \triangleq\left[\rho_{m, 0} \rho_{m, 1} \cdots \rho_{m, J-1}\right]$. For any $J \in \mathbb{N}$ and an arbitrary set of complex numbers $\left\{\rho_{m, j}\right\}_{j=0}^{J-1}$, the following holds:

$$
\mathbf{G}_{m} \mathbf{H}_{m}=\mathcal{H}_{m}\left(\boldsymbol{\rho}_{m}\right) \boldsymbol{\Theta}_{m}
$$

The choice of $\left\{\rho_{m, j}\right\}_{j=0}^{J-1}$ (which are also called signature points) is such that $\mathbf{G}_{m}$ eliminates MUI, as will be explained next. It will become apparent that the signature points need to be distinct.

Consider the interference from user $\mu \neq m$. From Fig. 2(c), it follows that the interfering signal $\mathbf{s}_{\mu}(n)$ passes through the concatenation of matrices

$$
\begin{aligned}
& \mathbf{G}_{m} \mathbf{H}_{\mu} \mathbf{C}_{\mu} \\
& \quad=\mathcal{H}_{\mu}\left(\boldsymbol{\rho}_{m}\right) \boldsymbol{\Theta}_{m} \mathbf{C}_{\mu}=\mathcal{H}_{\mu}\left(\boldsymbol{\rho}_{m}\right) \mathcal{C}_{\mu}\left(\boldsymbol{\rho}_{m}\right), \\
& \mathcal{C}_{\mu}\left(\boldsymbol{\rho}_{m}\right) \\
& \quad=\left[\begin{array}{cccc}
C_{\mu, 0}\left(\rho_{m, 0}\right) & C_{\mu, 1}\left(\rho_{m, 0}\right) & \cdots & C_{\mu, K-1}\left(\rho_{m, 0}\right) \\
C_{\mu, 0}\left(\rho_{m, 1}\right) & C_{\mu, 1}\left(\rho_{m, 1}\right) & \cdots & C_{\mu, K-1}\left(\rho_{m, 1}\right) \\
\vdots & \vdots & & \vdots \\
C_{\mu, 0}\left(\rho_{m, J-1}\right) & C_{\mu, 1}\left(\rho_{m, J-1}\right) & \cdots & C_{\mu, K-1}\left(\rho_{m, J-1}\right)
\end{array}\right] .
\end{aligned}
$$

The first equality in (7) is a consequence of (6). From (7), we see that in order to eliminate MUI, regardless of the channels, it suffices to choose $\left\{\rho_{m, j}\right\}_{m, j=0}^{M-1, J-1}$ such that

$C_{\mu, k}\left(\rho_{m, j}\right)=0, \forall m \neq \mu, \forall k \in[0, K-1], \forall j \in[0, J-1]$.

In practice, the signature points $\rho_{m, j}$ are often chosen to be uniformly spaced on the unit circle

$$
\rho_{m, l}=e^{j \frac{2 \pi(m+l M)}{M J}}, \quad 0 \leq l \leq J-1
$$

since this leads to fast Forier transform (FFT)-based AMOUR implementations having low complexity [3].

Equations (9) define $(M-1) J$ zeros of the polynomials $C_{m, k}(z)$. In addition to this, let $C_{m, k}(z)$ be such that

$$
C_{m, k}\left(\rho_{m, j}\right)=A_{m} \rho_{m, j}^{-k}
$$

where the multipliers $A_{m}$ introduce a simple power control for different users. At this point, the total number of constraints for each of the spreading polynomials is equal to $M J$. Recalling that the last $L$ samples of spreading codes are fixed to be zero, the minimum spreading code length is given by $P=M J+L$. Substituting (11) in (7) for $\mu=m$ and recalling (6), we have

$$
\mathbf{G}_{m} \mathbf{H}_{m} \mathbf{C}_{m}=A_{m} \underbrace{\left[\begin{array}{cccc}
1 & \rho_{m, 0}^{-1} & \cdots & \rho_{m, 0}^{-J+1} \\
1 & \rho_{m, 1}^{-1} & \cdots & \rho_{m, 1}^{-J+1} \\
\vdots & \vdots & & \vdots \\
1 & \rho_{m, J-1}^{-1} & \cdots & \rho_{m, J-1}^{-J+1}
\end{array}\right]}_{\mathbf{V}_{m}} \mathbf{H}_{m}
$$

where $\overline{\mathbf{H}}_{m}$ is the $J \times K$ north-west submatrix of $\mathbf{H}_{m}$.

In order to perform the channel equalization after MUI has been eliminated, we need to invert the matrix product $\mathbf{V}_{m} \overline{\mathbf{H}}_{m}$ in (12), which in turn needs to be of sufficient rank. From (7), with $\mu=m$, we conclude that (12) can be further written as a product of a diagonal matrix $\mathcal{H}_{m}\left(\boldsymbol{\rho}_{m}\right)$ and a $J \times K$ Vandermonde matrix $\mathcal{C}_{\mu}\left(\boldsymbol{\rho}_{m}\right)$. The second matrix $\mathcal{C}_{\mu}\left(\boldsymbol{\rho}_{m}\right)$ is invertible as long as $\left\{\rho_{m, j}\right\}$ are distinct. The rank of $\mathcal{H}_{m}\left(\boldsymbol{\rho}_{m}\right)$ can drop by at most $L$ only if all the zeros of $H_{m}(z)$ occur at the signature points $\rho_{m, j}$. Thus, the sufficient condition for the invertibility of (12) is $J=K+L$. In summary, the minimal system parameters are given by

$$
\begin{aligned}
& J=K, \quad(\text { known CSI }) \\
& J=K+L, \quad(\text { unknown CSI }) \quad \text { and } \\
& P=M J+L .
\end{aligned}
$$

In the limit when $K$ tends to infinity, the bandwidth expansion becomes

$$
\begin{aligned}
\text { BW expansion } & =\frac{P}{K} \\
& = \begin{cases}\frac{[M K+L]}{K} & \text { for known CSI } K \rightarrow \infty \\
\frac{[M(K+L)+L]}{K} & \text { unknown CSI } M .\end{cases}
\end{aligned}
$$

Since there are $M$ simultaneous transmitters in the system, this is the minimum possible bandwidth expansion.

From Fig. 2(c), it readily follows that (ignoring the noise)

$$
\hat{\mathbf{s}}_{m}(n)=A_{m} \boldsymbol{\Gamma}_{m} \mathbf{V}_{m}^{-1} \mathbf{V}_{m} \overline{\mathbf{H}}_{m} \mathbf{s}_{m}(n)=A_{m} \boldsymbol{\Gamma}_{m} \overline{\mathbf{H}}_{m} \mathbf{s}_{m}(n) .
$$

Therefore, $\Gamma_{m}$ can be chosen to eliminate the ISI in the absence of noise, and this would be a ZFE. For more details on this and alternative equalizers, see [3] and [4]. In the following, we consider the improvement of this conventional AMOUR system obtained by sampling the received continuous-time signal more densely than at the symbol-rate given by the transmitters.

\section{AMOUR WITH INTEGRAL OVERSAMPLING}

Fractionally spaced equalizers (FSEs) typically show an improvement in performance at the expense of more computations per unit time required at the receiver. FSEs with integral oversampling operate on a discrete-time signal obtained by sampling the received continuous-time signal $q$ times faster than at the transmission rate (thus the name fractionally spaced). Here, $q$ is 




Fig. 3. (a) Continuous-time model for the AMOUR system with integral oversampling. (b) Discrete-time equivalent drawing. (c) Polyphase representation for $q=2$.

assumed to be an integer greater than one. Our goal in this section is to introduce the benefits of FSEs in the ISI suppression, without violating the conditions for perfect MUI cancellation, irrespective of the uplink channels. As will be clear shortly, this is entirely achieved through the use of the FSAMOUR system, introduced in the following.

In order to develop the discrete-time equivalent structure for the AMOUR system with integral oversampling at the receiver, we consider the continuous-time AMOUR system with an FSE shown in Fig. 3(a). Let $T$ be defined as the symbol spacing at the output of the transmitter [signal $u_{m}(n)$ in Fig. 3(a)]. Working backward, we conclude that the rate of the blocked signal $\mathbf{s}_{m}(n)$ is $P$ times lower, i.e., $1 / P T$. Since $\mathbf{s}_{m}(n)$ is obtained by parsing the information sequence $s_{m}(n)$ into blocks of length $K$, as shown in Fig. 2(a), we conclude that the corresponding data rate of $s_{m}(n)$ at the transmitter is $K / P T$.

Each of the transmitted discrete signals $u_{m}(n)$ are first converted into analog signals and passed through a pulse-shaping filter. The combined effect of the reconstruction filter from the D/A converter, the pulse shaping filter, and the continuous time uplink channel followed by the receive filters is referred to as the equivalent channel and is denoted by $h_{c}(t)$. After passing through the equivalent channel, the signal is corrupted by the additive noise and interference from other users. The received waveform $x_{c}(t)$ is sampled at $q$ times the rate at the output of the transmitter [see Fig. 3(a)]. The sequence $x_{m}(n)$ with rate $q / T$ enters the fractionally spaced equalizer that operates at the correspondingly higher rate. Accompanied with the equalization process, some rate reduction also needs to take place at the receiver so that the sequence $\hat{s}_{m}(n)$ at the decision device has exactly the same rate $K / P T$ as the starting information sequence.

Now, we derive the discrete-time equivalent of the oversampled system from Fig. 3(a). Consider the received sequence $x_{m}(n)$ in the absence of noise and MUI. We can see that

$$
x_{m}(n)=x_{c}\left(n \frac{T}{q}\right)=\sum_{k=-\infty}^{\infty} u_{m}(k) h_{c}\left(n \frac{T}{q}-k T\right) .
$$

Defining the discrete time sequence $h_{m}^{(q)}(n) \triangleq h_{c}(n T / q)$, which is nothing but the waveform $h_{c}(t)$ sampled $q$ times more densely than at integers, we have

$$
x_{m}(n)=\sum_{k=-\infty}^{\infty} u_{m}(k) h_{m}^{(q)}(n-k q) .
$$

This is shown in Fig. 3(b), where the noise and MUI, which were continuous functions of time in Fig. 3(a), now need to be modified (by appropriate sampling). Notice that although the discrete-time equivalent structure incorporates the upsampling by $q$ at the output of the transmitters, this does not result in any bandwidth expansion since the physical structure is still given in Fig. 3(a). Our goal in this section is to design the block in Fig. 3(b) labeled "equalization and rate reduction." In the following, we introduce one possible solution that preserves the MUI cancellation property, as it was described in Section II, yet provides additional flexibility when it comes to the ISI elimination part. For simplicity, in what follows, we assume $q=2$; however, it is easy to show that a similar design procedure follows through for any integer $q$.

Oversampling by $q=2$. First, we redraw the structure in Fig. 3(b), as shown in Fig. 3(c). Here, $H_{m, 0}(z)$ and $H_{m, 1}(z)$ are the Type-1 polyphase components [14] of the oversampled filter $H_{m}^{(2)}(z)$. In other words

$$
H_{m}^{(2)}(z)=H_{m, 0}\left(z^{2}\right)+z^{-1} H_{m, 1}\left(z^{2}\right)
$$

In Fig. 3(c), we also moved the additive noise and interference past the delay and upsamplers by splitting them into appropriate polyphase components in a fashion similar to (16). Before we proceed with the design of the FSAMOUR receiver, we recall that the construction of the spreading codes $\left\{C_{m, k}(z)\right\}$ and the receive filters $\left\{G_{m, j}(z)\right\}$ in Section II ensured the elimination of MUI, regardless of the propagation channels, as long as their delay spreads are bounded by $L$. Returning to Fig. 3(c) in view 


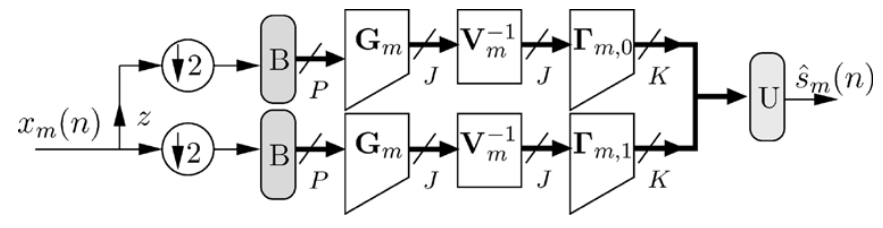

Fig. 4. Proposed form of the equalizer with rate reduction.

of (16), we notice that $H_{m, 0}(z)$ is nothing but the original integer-sampled channel $H_{m}(z)$. In addition, each of the subchannels $H_{m, i}(z)$ can have the order at most equal to the order of $H_{m}(z)$, i.e., the maximum order of $H_{m, i}(z)$ is $L$. Moreover, each of the $q$ polyphase components of MUI shown in Fig. 3(c) is obtained by passing the interfering signals $u_{\mu}(n)$ through the corresponding channel polyphase components $H_{\mu, i}(z)$. From the discussion in Section II, we know how to eliminate each of these MUI components separately. Therefore, our approach in the equalizer design will be to keep these polyphase channels separate, perform the MUI cancellation in each of them, and combine the results to obtain the MUI-free signal received from user $m$. This is achieved by the structure shown in Fig. 4 .

The received oversampled signal is first divided into the Type- 2 polyphase components (a total of $q$ polyphase components for oversampling by $q$ ). This operation assures that in each of the equalizer branches the symbol rate is equal to $1 / T$. At the same time, each branch contains only one polyphase component of the desired signal and MUI from Fig. 3(c). These polyphase components are next passed through a system that resembles the conventional AMOUR receiver structure from Fig. 2(a). Notice one difference: While the matrices $\mathbf{G}_{m}$ and $\mathbf{V}_{m}^{-1}$ are kept the same as before, the matrices for ISI mitigation $\boldsymbol{\Gamma}_{m, i}$ are different in each branch, and their outputs are combined, forming the information signal estimate $\hat{s}_{m}(n)$. Careful observation confirms that the output symbol rate is equal to $K / P T$, precisely as desired.

In order to further investigate the properties of the proposed solution, we show the complete FSAMOUR system in terms of the equivalent matrix building blocks in Fig. 5(a). The effect of the oversampling followed by the receiver structure with $q$ branches is equivalent to receiving $q$ copies of each transmitted signal but after going through different multipath fading channels $H_{m, i}(z)$. This temporal diversity in the received signal is obviously beneficial for the equalization process, as will be demonstrated in Section III-A. As mentioned previously, MUI elimination in AMOUR systems does not depend on the uplink channels as long as their order is upper bounded by $L$, and this is why the proposed FSAMOUR system eliminates MUI in each branch of Fig. 5(a). Notice that the length restrictions on $P$ and $J$ for MUI elimination remain the same as in Section II.

Repeating the matrix manipulations similar to those demonstrated in Section II, but this time in each branch separately, we conclude that the equivalent FSAMOUR system is shown in Fig. 5(b). Lower triangular Toeplitz matrices $\overline{\mathbf{H}}_{m, i}$ here correspond to different polyphase components of the oversampled channel. Noise vectors $\mathbf{e}_{i}(n)$ are obtained by appropriately blocking and filtering the noise from Fig. 5(a). As in [3] and [4], the equalizer $\boldsymbol{\Gamma}_{m}=\left[\boldsymbol{\Gamma}_{m, 0} \boldsymbol{\Gamma}_{m, 1}\right]$ can be constructed as a
RAKE, zero-forcing, or MMSE receiver corresponding to the transmitter $\overline{\mathbf{H}}_{m}=\left[\overline{\mathbf{H}}_{m, 0}^{T} \overline{\mathbf{H}}_{m, 1}^{T}\right]^{T}$ :

$$
\begin{aligned}
\boldsymbol{\Gamma}_{m}^{\text {(rake) }} & =\overline{\mathbf{H}}_{m}^{\dagger} \\
\Gamma_{m}^{(\mathrm{zfe})} & =\left(\overline{\mathbf{H}}_{m}^{\dagger} \overline{\mathbf{H}}_{m}\right)^{-1} \overline{\mathbf{H}}_{m}^{\dagger}(\text { pseudo-inverse }) \\
\boldsymbol{\Gamma}_{m}^{(\text {mmse })} & =\mathcal{R}_{\mathbf{s s}} \overline{\mathbf{H}}_{m}^{\dagger}\left(\mathcal{R}_{\text {ee }}+\overline{\mathbf{H}}_{m} \mathcal{R}_{\mathbf{s s}} \overline{\mathbf{H}}_{m}^{\dagger}\right)^{-1}
\end{aligned}
$$

where $\mathcal{R}_{\mathrm{ss}}$ and $\mathcal{R}_{\text {ee }}$ represent the autocorrelation matrices of the signal $\mathbf{s}_{m}(n)$ and noise $\mathbf{e}(n) \triangleq\left[\mathbf{e}_{0}^{T}(n) \mathbf{e}_{1}^{T}(n)\right]^{T}$ processes, respectively. See Fig. 5(b).

The improvement in performance over the conventional AMOUR system comes as a result of having more degrees of freedom in the construction of equalizers, namely $q J-K$ more rows than columns in FSAMOUR compared to $J-K$ in AMOUR. Another way to appreciate this additional freedom in the ZFE design is as follows. In the AMOUR systems, the construction of ZFEs amounts to finding $\boldsymbol{\Gamma}_{m}$, as in (13), such that $\boldsymbol{\Gamma}_{m} \hat{\mathbf{H}}_{m}=\mathbf{I}_{K}$; in other words, $\boldsymbol{\Gamma}_{m}$ is a left inverse of $\hat{\mathbf{H}}_{m}$. On the other hand, referring to Fig. 5(b), we conclude that the ZFEs in the FSAMOUR systems need to satisfy

$$
\Gamma_{m, 0} \hat{\mathbf{H}}_{m, 0}+\boldsymbol{\Gamma}_{m, 1} \hat{\mathbf{H}}_{m, 1}=\mathbf{I}_{K}
$$

thus providing more possibilities for the design of $\boldsymbol{\Gamma}_{m, i}$. In addition to all this, the performance of the zero-forcing solutions can be further improved by noticing that left inverses of $\overline{\mathbf{H}}_{m}$ are not unique. In the following subsection, we derive the best ZFE for a given FSAMOUR system with the oversampling factor $q$. This optimal solution corresponds to taking advantage of the $q J-K$ degrees of freedom present in the equalizer design.

\section{A. Optimal FSAMOUR ZFE}

Consider the equivalent FSAMOUR system given in Fig. 6(a). It corresponds to the system shown in Fig. 5(b) with one difference; namely, the block-equalizer is allowed to have memory. In the following, we investigate the case of ZFE, which corresponds to having $\hat{\mathbf{s}}_{m}(n)=\mathbf{s}_{m}(n)$ in the absence of noise. Obviously, this is achieved if and only if $\boldsymbol{\Gamma}_{m}(z)$ is a left inverse of $\overline{\mathbf{H}}_{m}$. Under the conditions on $P$ and $J$ described in Section II, this inverse exists. Moreover, the fact that $\overline{\mathbf{H}}_{m}$ is tall implies that this inverse is not unique. Our goal is to find the left inverse $\boldsymbol{\Gamma}_{m}(z)$, as in Fig. 6(a), of a given order that will minimize the noise power at the output, i.e., minimize the power of $\hat{\mathbf{s}}_{m}(n)$, given that $\mathbf{s}_{m}(n)=0$. The equalizer design described here is closely related to the solution of a similar problem presented in [21]. One difference is that the combined transmitter/channel matrix $\overline{\mathbf{H}}_{m}$ in Fig. 6(a) is constant, so we use its singular value decomposition [5] instead of a Smith-form decomposition, as in [21].

The tall rectangular matrix $\overline{\mathbf{H}}_{m}$ can be decomposed as [5]

$$
\overline{\mathbf{H}}_{m}=\mathbf{U}_{m} \cdot\left[\begin{array}{c}
\boldsymbol{\Sigma}_{m} \\
\mathbf{0}
\end{array}\right] \cdot \mathbf{V}_{m}
$$

where $\mathbf{U}_{m}$ and $\mathbf{V}_{m}$ are $q J \times q J$ and $K \times K$ unitary matrices, respectively, and $\Sigma_{m}$ is a $K \times K$ diagonal matrix of singular 


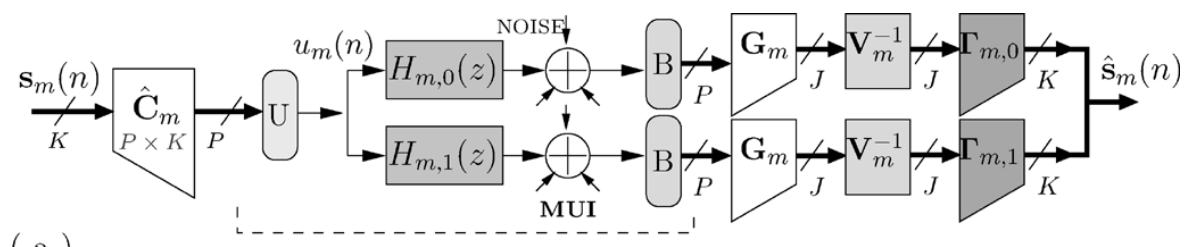

( a )

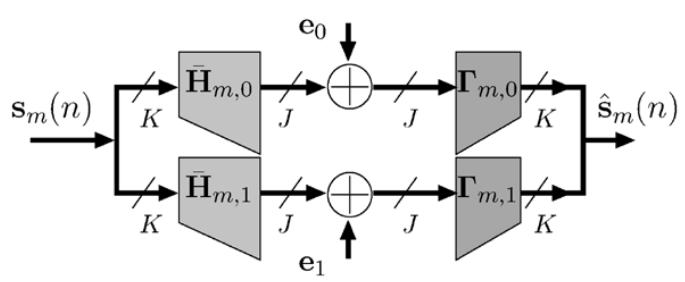

Fig. 5. (a) Proposed overall structure of the FSAMOUR system. (b) Simplified equivalent structure for ISI suppression.

values. Since we assumed $\overline{\mathbf{H}}_{m}$ has rank $K$, it follows that $\boldsymbol{\Sigma}_{m}$ is invertible. It can be seen from (18) that the most general form of a left inverse of $\overline{\mathbf{H}}_{m}$ is given by

$$
\boldsymbol{\Gamma}_{m}(z)=\mathbf{V}_{m}^{\dagger}\left[\boldsymbol{\Sigma}_{m}^{-1} \mathbf{A}_{m}(z)\right] \mathbf{U}_{m}^{\dagger}
$$

where $\mathbf{A}_{m}(z)$ is an arbitrary $K \times(q J-K)$ polynomial matrix and represents a handle on the degrees of freedom in the design of $\boldsymbol{\Gamma}_{m}(z)$. Defining the $K \times q J,(q J-K) \times q J$, and $K \times(q J-$ $K)$ matrices $\mathbf{D}_{0}, \mathbf{D}_{1}$, and $\mathbf{B}_{m}(z)$, respectively, as

$$
\left[\begin{array}{l}
\mathbf{D}_{0} \\
\mathbf{D}_{1}
\end{array}\right] \triangleq \mathbf{U}_{m}^{\dagger}, \quad \text { and } \quad \mathbf{B}_{m}(z) \triangleq \mathbf{V}_{m}^{\dagger} \cdot \mathbf{A}_{m}(z)
$$

(19) can be rewritten as [see Fig. 6(b)]

$$
\Gamma_{m}(z)=\mathbf{V}_{m}^{\dagger} \Sigma_{m}^{-1} \cdot \mathbf{D}_{0}+\mathbf{B}_{m}(z) \cdot \mathbf{D}_{1} .
$$

Since there is a one-to-one correspondence (20) between the matrices $\mathbf{A}_{m}(z)$ and $\mathbf{B}_{m}(z)$, the design objective becomes that of finding the $\mathbf{B}_{m}(z)$ of a fixed-order $N_{b}-1$, which is given by its impulse response

$$
\mathbf{B}_{m}(z)=\sum_{n=0}^{N_{b}-1} \mathbf{B}_{m, n} z^{-n},
$$

that minimizes the noise power $E\left\{\hat{\mathbf{e}}_{m}^{\dagger} \hat{\mathbf{e}}_{m}\right\} / K$ at the output of Fig. 6(b). The operator $E\{\cdot\}$ denotes the expected value. From Fig. 6(b), it is evident that the optimal $\mathbf{B}_{m}(z)$ in this context is nothing but a linear estimator of a vector random process $\mathbf{u}(n)$ given $\mathbf{v}(n)$. The solution is well-known [11] and is given by

$$
\left[\mathbf{B}_{m, 0} \mathbf{B}_{m, 1} \cdots \mathbf{B}_{m, N_{b}-1}\right] \triangleq \mathcal{B}=-E\left\{\mathbf{u}(n) \mathcal{V}^{\dagger}(n)\right\} \cdot \mathcal{R}_{\mathcal{V} \mathcal{V}}^{-1}
$$

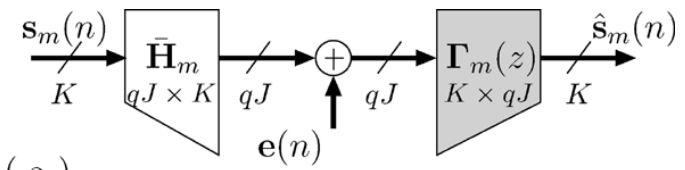

( a )

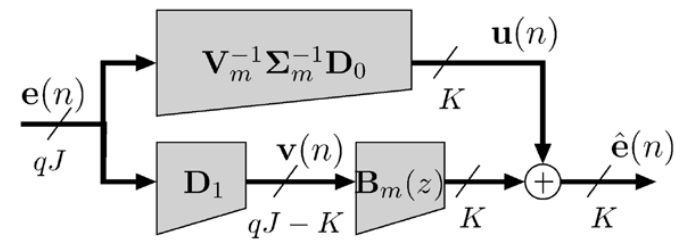

( b )

Fig. 6. (a) Equivalent FSAMOUR system. (b) ZFE structure with noise input.

where $\mathcal{V}(n) \triangleq\left[\mathbf{v}^{T}(n) \mathbf{v}^{T}(n-1) \cdots \mathbf{v}^{T}\left(n-N_{B}+1\right)\right]^{T}$, and $\mathcal{R}_{\mathcal{V V}}$ is its autocorrelation matrix. Next, we rewrite the solution (23) in terms of the noise statistics, namely, its $q J \times q J$ crosscorrelation matrices $\mathcal{R}_{\mathbf{e e}}(k) \triangleq E\left\{\mathbf{e}_{m}(n) \mathbf{e}_{m}^{\dagger}(n-k)\right\}$. First note that we have (24), shown at the bottom of the page. Similarly, we can rewrite

$$
\begin{aligned}
& E\left\{\mathbf{u}(n) \mathcal{V}^{\dagger}(n)\right\}=\mathbf{V}_{m}^{\dagger} \cdot \boldsymbol{\Sigma}_{m}^{-1} \cdot \mathbf{D}_{0} \\
& \cdot\left[\mathcal{R}_{\mathbf{e e}}(0) \mathbf{D}_{1}^{\dagger} \mathcal{R}_{\mathbf{e e}}(1) \mathbf{D}_{1}^{\dagger} \cdots \mathcal{R}_{\mathbf{e e}}\left(N_{b}-1\right) \mathbf{D}_{1}^{\dagger}\right]
\end{aligned}
$$

For sufficiently large input block size $q J$, it is often safe to assume that the noise is uncorrelated across different blocks; in other words, $\mathcal{R}_{\text {ee }}(k)=\mathbf{0}$ for $k \neq 0$. In this important special case, the optimal $\mathbf{B}_{m}(z)$ is a constant, namely

$$
\mathbf{B}_{m}(z)=\mathbf{B}_{m, 0}=-\mathbf{V}_{m}^{\dagger} \Sigma_{m}^{-1} \mathbf{D}_{0} \mathcal{R}_{\mathbf{e e}}(0) \mathbf{D}_{1}^{\dagger}\left(\mathbf{D}_{1} \mathcal{R}_{\mathbf{e e}}(0) \mathbf{D}_{1}^{\dagger}\right)^{-1}
$$

$$
\mathcal{R}_{\mathcal{V} \mathcal{V}}=\left[\begin{array}{cccc}
\mathbf{D}_{1} \mathcal{R}_{\mathbf{e e}}(0) \mathbf{D}_{1}^{\dagger} & \mathbf{D}_{1} \mathcal{R}_{\mathbf{e e}}(1) \mathbf{D}_{1}^{\dagger} & \cdots & \mathbf{D}_{1} \mathcal{R}_{\mathbf{e e}}\left(N_{b}-1\right) \mathbf{D}_{1}^{\dagger} \\
\mathbf{D}_{1} \mathcal{R}_{\mathbf{e e}}(1) \mathbf{D}_{1}^{\dagger} & \mathbf{D}_{1} \mathcal{R}_{\mathbf{e e}}(0) \mathbf{D}_{1}^{\dagger} & \cdots & \mathbf{D}_{1} \mathcal{R}_{\mathbf{e e}}\left(N_{b}-2\right) \mathbf{D}_{1}^{\dagger} \\
\vdots & \vdots & \ddots & \vdots \\
\mathbf{D}_{1} \mathcal{R}_{\mathbf{e e}}\left(N_{b}-1\right) \mathbf{D}_{1}^{\dagger} & \mathbf{D}_{1} \mathcal{R}_{\mathbf{e e}}\left(N_{b}-2\right) \mathbf{D}_{1}^{\dagger} & \cdots & \mathbf{D}_{1} \mathcal{R}_{\mathbf{e e}}(0) \mathbf{D}_{1}^{\dagger}
\end{array}\right]
$$


From (26) and (21), we get the optimal form of a ZFE

$$
\begin{aligned}
\boldsymbol{\Gamma}_{m}^{(\mathrm{opt})}=\mathbf{V}_{m}^{\dagger} \boldsymbol{\Sigma}_{m}^{-1}\left[\mathbf{I}_{K}-\right. & \mathbf{D}_{0} \mathcal{R}_{\mathbf{e e}}(0) \mathbf{D}_{1}^{\dagger} \\
& \left.\times\left(\mathbf{D}_{1} \mathcal{R}_{\mathbf{e e}}(0) \mathbf{D}_{1}^{\dagger}\right)^{-1}\right] \mathbf{U}_{m}^{\dagger} .
\end{aligned}
$$

Another important special case occurs when the noise samples at the input of the receiver are i.i.d. It is important to notice here that $\mathbf{e}(n)$ in Figs. 5 and 6 is obtained by passing the input noise through a bank of $q$ receiver front ends $\mathbf{V}_{m}^{-1} \mathbf{G}_{m}$. Therefore, the noise autocorrelation matrix $\mathcal{R}_{\mathbf{e e}}(0)$ is not likely to be a scaled identity. Instead, in this case, we have

$$
\mathcal{R}_{\mathrm{ee}}(k)=\delta_{k} \cdot \operatorname{diag}_{0 \leq z \leq q-1}\left\{\sigma_{z}^{2} \cdot \mathbf{V}_{m}^{-1} \mathbf{G}_{m} \mathbf{G}_{m}^{\dagger} \mathbf{V}_{m}^{-\dagger}\right\}
$$

which is a $q J \times q P$ block-diagonal matrix, with noise variances $\sigma_{z}^{2}$ corresponding to different signal polyphase components. Starting from (4) and (12), we can readily verify that for large values of $M, \mathbf{V}_{m}^{-1} \mathbf{G}_{m} \mathbf{G}_{m}^{\dagger} \mathbf{V}_{m}^{-\dagger} \approx M \cdot \mathbf{I}_{J}$. Therefore, in the case of white channel noise and no oversampling in a system with many users, the optimal ZFE from (27) becomes

$$
\Gamma_{m}^{\text {(white noise) }}=\mathbf{V}_{m}^{\dagger}\left[\Sigma_{m}^{-1} \mathbf{0}\right] \mathbf{U}_{m}^{\dagger} .
$$

This follows since $\mathbf{D}_{0} \mathbf{D}_{1}^{\dagger}=\mathbf{0}$ and $\mathcal{R}_{\text {ee }}(k) \approx \delta_{k} \cdot \sigma_{k}^{2} \cdot \mathbf{I}$.

At this point, we would like to make a distinction between the optimal ZFEs in the AMOUR and FSAMOUR systems. From the derivations presented in this subsection, it is evident that the optimal ZFEs can be constructed in a traditional AMOUR system of [3], [4], and it is to be expected that this solution would perform better than the ordinary ZFE based on the matrix pseudo-inverse similar to (17). However, in the following, we show that if the channel noise in Fig. 3(a) is i.i.d., then any optimization of ZFEs in AMOUR systems will not improve their performance. This is not true for fractionally spaced AMOUR systems since the noise samples in vectors $\mathbf{e}_{0}(n)$ and $\mathbf{e}_{1}(n)$ in Fig. 6(b) need not have the same variances, although they remain independent. This is due to the fact that $\mathbf{e}_{0}(n)$ and $\mathbf{e}_{1}(n)$ correspond to signals received through different polyphase components of the channel. Consequently, in the FSAMOUR case, the noise autocorrelation matrices $\mathcal{R}_{\text {ee }}(0)$ appearing in (27) are not given by scaled identity matrices, and (29) does not correspond to the optimal solution. Now, let us compare the optimal ZFE in the AMOUR system for the white noise (29) to the corresponding zero-forcing solution given in (17). The result is summarized as follows.

Proposition 1: Pseudo-inverse is the optimal AMOUR ZF SSE if the noise is white.

Comment: This result is indeed well known. See [7] for a detailed treatment of various equalizers in a traditional CDMA system. For completeness, in the following, we give a short proof of Proposition 1.

Proof: Starting from the traditional ZFE $\Gamma_{m}^{(\mathrm{zfe})}$, we have

$$
\begin{aligned}
\boldsymbol{\Gamma}_{m}^{(\mathrm{zfe})} & =\left(\overline{\mathbf{H}}_{m}^{\dagger} \overline{\mathbf{H}}_{m}\right)^{-1} \overline{\mathbf{H}}_{m}^{\dagger} \\
& =\left(\mathbf{V}_{m}^{\dagger}\left[\boldsymbol{\Sigma}_{m}^{\dagger} \mathbf{0}\right] \mathbf{U}_{m}^{\dagger} \mathbf{U}_{m}\left[\begin{array}{c}
\left.\boldsymbol{\Sigma}_{m}\right] \\
\mathbf{0}
\end{array}\right] \mathbf{V}_{m}\right)^{-1} \mathbf{V}_{m}^{\dagger}\left[\boldsymbol{\Sigma}_{m}^{\dagger} \mathbf{0}\right] \mathbf{U}_{m}^{\dagger} \\
& =\mathbf{V}_{m}^{\dagger}\left[\boldsymbol{\Sigma}_{m}^{-1} \mathbf{0}\right] \mathbf{U}_{m}^{\dagger} \\
& =\boldsymbol{\Gamma}_{m}^{\text {(white noise) }}
\end{aligned}
$$



Fig. 7. Probability of error as a function of SNR in AMOUR and FSAMOUR systems.

A more insightful way to look at the result from Proposition 1 is that there is nothing to be gained by using the optimal solution if there is no oversampling at the receiver. In contrast to this, using the optimal ZFEs in FSAMOUR systems leads to a noticeable improvement in performance over the simple pseudo-inverses, as is demonstrated in Section III-B. Finally, note that an alternative to using the equalizer (27) would be to apply pre-whitening filters followed by equalizers from (29).

\section{B. Performance Evaluation}

In this subsection, we compare the performance of the conventional (SSE) AMOUR described in Section II and the FSAMOUR system from Section III with oversampling ratio $q=2$. System parameters in the experiment were given by $K=12$ and $M=4$, while $J$ and $P$ were chosen to be the minimum for the guaranteed existence of channel ZFEs, as explained in Section II. The performance results were obtained by averaging over 30 multipath channel realizations. The equivalent channel was modeled as a combination of a raised cosine (constant part in the transmitter and the receiver) and a randomly chosen short multipath channel. The resulting half-integer sampled, channel impulse responses $h_{m}^{(2)}(n)$ were of the 11th order. The equivalent, integer-spaced channels were obtained by keeping the even samples and are of order $L=5$.

The channel noise, which was originally AWGN, was colored by the square-root raised-cosine at the receiver. The signal-tonoise ratio (SNR) was measured after sampling at the entrance of the receiver [point $x_{m}(n)$ in Fig. 3(a)]. Notice that SNR does not depend on the oversampling ratio $q$ as long as the signal and the noise are stationary. The performance curves are shown in Fig. 7. The acronyms "SSE" and "FSE" represent AMOUR and FSAMOUR systems, whereas the suffixes "ZF," "MMSE," and "OPT" correspond to zero-forcing, minimum mean-squared error, and optimal ZFE solutions, respectively. There are several important observations that can be made from these results.

- The overall performance of AMOUR systems is significantly improved by signal oversampling at the receiver. 


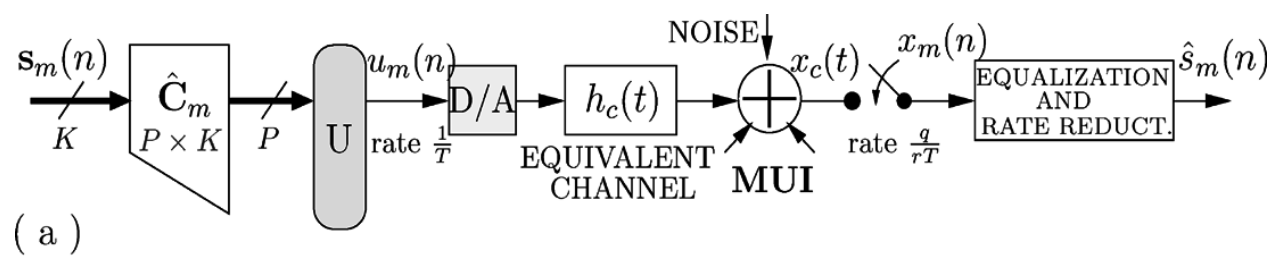

(a)

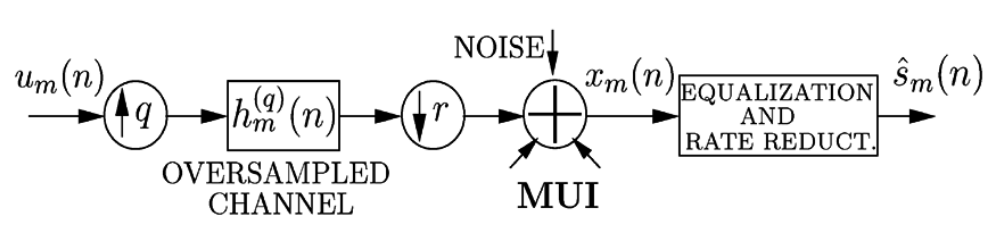

Fig. 8. (a) Continuous-time model for the AMOUR system with fractional oversampling ratio $q / r$. (b) Equivalent discrete-time system.

-

The performance of ZFEs in FSAMOUR systems can be further improved by about $0.4 \mathrm{~dB}$ by using the optimal equalizers that exploit the redundancy in ZFE design, as described in Section III-A. This is due to the fact that the optimal solution is given by (27) rather than (29). As explained previously, the same does not hold for AMOUR systems.

- The performance of the optimal ZFEs in FSAMOUR systems is almost identical to the performance of the optimal $^{1}$ MMSE equalizers. Thus, there is practically no loss in performance as a result of using the optimal ZFE given by (27) instead of the MMSE equalizer (17). The advantages of using a ZFE become evident by comparing the expressions (27) and (17). As opposed to the MMSE solution $\Gamma_{m}^{(\mathrm{mmse})}$, ZFE $\Gamma_{m}^{(\text {opt })}$ does not require the knowledge of the signal statistics $\mathcal{R}_{\mathrm{ss}}$, and if the noise is white and stationary, the solution $\boldsymbol{\Gamma}_{m}^{(\mathrm{opt})}$ is independent of the noise variance, which plays a significant role in the corresponding MMSE solution (17). More detailed analysis of the mentioned advantages can be found in [20].

- Even though the noise was colored, a simple pseudoinverse happens to yield an almost identical performance as the MMSE equalizer and is therefore the optimal ZFE in AMOUR systems with no oversampling.

In the next section, we introduce the modification of the idea of the integral oversampling of the received signal to a more general case when the amount of oversampling is a rational number.

\section{AMOUR WITH FRACTIONAL OVERSAMPLING}

While FSAMOUR systems with the integral oversampling can lead to significant improvement in performance compared to traditional AMOUR systems, the notion of oversampling the received CDMA signal might be less popular due to very high data rates of the transmitted CDMA signals. According to the scenario of integral oversampling, the data rates at the receiver are at least twice as high as the rates at the transmitter, which makes them prohibitively high for most sophisticated equalization techniques. In this section, we explore the consequences of sampling the continuous-time received signal $x_{c}(t)$ in Fig. 3(a) at a rate that is higher than the symbol rate $1 / T$ by a frac-

\footnotetext{
${ }^{1}$ The MMSE equalizer is the optimal solution in terms of minimizing the energy of the error signal at the receiver for the fixed system parameters.
}

tional amount. To be more precise, suppose the amount of oversampling is $q / r$, where $q$ and $r$ are coprime integers satisfying $q>r$. If $q=r+1$ for high values of $r$, the data rate at the receiver becomes almost identical to the one at the transmitter, which is rather advantageous from the implementational point of view. It will soon become evident that the case when $q$ and $r$ share a common divisor can easily be reduced to the case of coprime factors. This said, it appears that the discussion from the previous section is redundant since it simply corresponds to fractional oversampling with $r=1$. However, it is instructive to consider the integer case separately since it is easier to analyze and provides some important insights.

Consider Fig. 3(a), and suppose $x_{c}(t)$ has been sampled at rate $q / r$. This situation is shown in Fig. 8(a). Performing the analysis that is very similar to the one in Section III, we can easily show that in this case, we have

$$
x_{m}(n)=\sum_{k=-\infty}^{\infty} u_{m}(k) h_{m}^{(q)}(n r-k q) .
$$

This is shown in Fig. 8(b), with appropriate modification of the noise from Fig. 8(a) and with $h_{m}^{(q)}(n)$ denoting $h_{c}(n T / q)$, just as it did in the case of integer oversampling.

The structure shown in Fig. 8(b) consisting of an expander by $q$, filter $H_{m}^{(q)}(z)$, and a decimator by $r$ has been studied extensively in [18]-[20]. It has been shown in [20] that without loss of generality, we can assume that $q$ and $r$ are coprime in such structures. Namely, if $p$ was a nontrivial greatest common divisor of $q$ and $r$ such that $q=q^{\prime} \cdot p$ and $r=r^{\prime} \cdot p$, with $q^{\prime}$ and $r^{\prime}$ mutually coprime, then the structure is equivalent to the one with $q$ replaced by $q^{\prime}, r$ replaced by $r^{\prime}$, and the new filter corresponding to the zeroth $p$-fold polyphase component [14] of $H_{m}^{(q)}(z)$.

Now, we are ready for the problem of multiuser communications with the rational oversampling ratio of $q / r$. The analysis of the fractionally oversampled FSAMOUR systems will turn out to be somewhat similar to the discussion in Section II, and in order to make the presentation more accessible, we have grouped the most important steps into separate subsections. One noticeable difference with respect to the material from Section II is that in this section, we will mostly deal with larger, block matrices. This comes as a consequence of a result on fractionally sampled channel responses, which was presented in a recent paper on fractional biorthogonal partners [20]. 


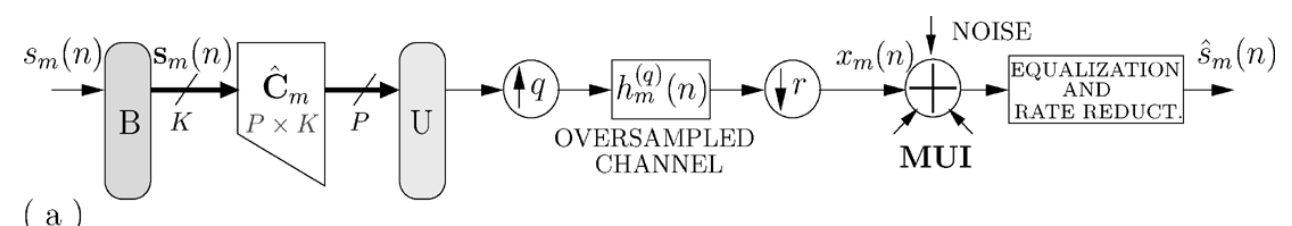

( a )

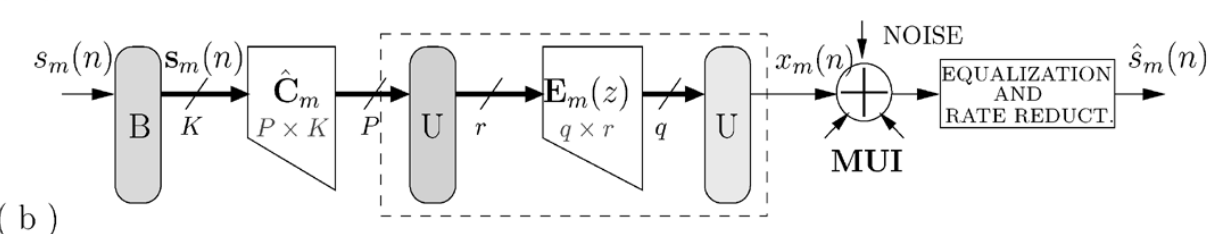

( b )



(c) $\quad \hat{\mathbf{E}}_{m}(z)$

Fig. 9. (a) Discrete-time model for the FSAMOUR system with the oversampling ratio $q / r$. (b) Equivalent drawing. (c) Redrawing a block from (b).

\section{A. Writing the Fractionally Sampled Channel as a Block Convolution}

Combining the elements from Figs. 8(a) and (b), we conclude that the discrete-time equivalent scheme of the FSAMOUR system with the oversampling ratio $q / r$ is shown in Fig. 9(a). It has been established in [20] that the operation of filtering by $H_{m}^{(q)}(z)$ surrounded by an expander and a decimator, as it appears in Fig. 9(a), is equivalent to blocking the signal, passing it through a $q \times r$ matrix transfer function $\mathbf{E}_{m}(z)$, and then unblocking it. This equivalent structure is employed in Fig. 9(b). The unblocking element of a darker shade represents the "incomplete" unblocking, i.e., it converts a sequence of blocks of length $P$ into a higher rate sequence of blocks of length $r$. In other words, it can be thought of as the unblocking of a length- $P$ vector sequence into a scalar sequence, followed by the blocking of the obtained scalar signal into a length- $r$ vector signal. Here, for simplicity, we assumed $r$ divides $P$; however, this condition is unnecessary for the above definition to hold, and we return to this point later.

The relation between the filter $H_{m}^{(q)}(z)$ and the corresponding matrix $\mathbf{E}_{m}(z)$ is rather complicated and is introduced in the following. First, let us write $H_{m}^{(q)}(z)$ in terms of its Type- $2 q$-fold polyphase components

$$
H_{m}^{(q)}(z)=\sum_{k=0}^{q-1} H_{m, k}\left(z^{q}\right) z^{k}
$$

Next, recall from the Euclid's algorithm that since $q$ and $r$ are mutually coprime, there exist $Q, R \in \mathbb{Z}$ such that

$$
q Q+r R=1
$$

Let us define the filters $P_{m, k}(z)$ and their Type- $1 r$-fold polyphase components $E_{k, l}(z)$ as

$P_{m, k}(z) \triangleq z^{k Q} H_{m, k}(z)=\sum_{l=0}^{r-1} E_{k, l}\left(z^{r}\right) z^{-l}$, for $0 \leq k \leq q-1$.

Then, it can be shown [20] that the equivalent matrix transfer function $\mathbf{E}_{m}(z)$ is given by

$$
\mathbf{E}_{m}(z)=\left[\begin{array}{cccc}
E_{0,0}(z) & E_{0,1}(z) & \cdots & E_{0, r-1}(z) \\
E_{1,0}(z) & E_{1,1}(z) & \cdots & E_{1, r-1}(z) \\
\vdots & \vdots & & \vdots \\
E_{q-1,0}(z) & E_{q-1,1}(z) & \cdots & E_{q-1, r-1}(z)
\end{array}\right] .
$$

Now, consider the block surrounded by a dashed line in Fig. 9(b). This can trivially be redrawn as in Fig. 9(c). The denoted $\bar{P} \times P$ transfer function $\hat{\mathbf{E}}_{m}(z)$ is the block pseudo-circulant in (36), shown at the bottom of the page. The $q \times r$ blocks $\mathbf{E}_{n}$, for $0 \leq n \leq N$ in (36), represent the impulse response of $\mathbf{E}_{m}(z)$, while $N$ is the order of the matrix polynomial and it depends on the choice of $r$ and on the maximum channel order $L$. This issue will be revisited shortly. It is implicitly assumed

$$
\hat{\mathbf{E}}_{m}(z)=\left[\begin{array}{cccccccc}
\mathbf{E}_{0} & \mathbf{0} & \cdots & \mathbf{0} & z^{-1} \mathbf{E}_{N} & z^{-1} \mathbf{E}_{N-1} & \cdots & z^{-1} \mathbf{E}_{1} \\
\mathbf{E}_{1} & \mathbf{E}_{0} & \cdots & \mathbf{0} & \mathbf{0} & z^{-1} \mathbf{E}_{N} & \cdots & z^{-1} \mathbf{E}_{2} \\
\vdots & \vdots & \ddots & \vdots & \vdots & \vdots & \ddots & \vdots \\
\mathbf{E}_{N} & \mathbf{E}_{N-1} & \cdots & \mathbf{E}_{0} & \mathbf{0} & \mathbf{0} & \cdots & \mathbf{0} \\
\mathbf{0} & \mathbf{E}_{N} & \cdots & \mathbf{E}_{1} & \mathbf{E}_{0} & \mathbf{0} & \cdots & \mathbf{0} \\
\vdots & \vdots & \ddots & \vdots & \vdots & \vdots & & \vdots \\
\mathbf{0} & \mathbf{0} & \cdots & \mathbf{E}_{N} & \mathbf{E}_{N-1} & \mathbf{E}_{N-2} & \cdots & \mathbf{E}_{0}
\end{array}\right]
$$


in (36) that $q$ divides $\bar{P}$. For arbitrary values of $r$ and $q$, we can write

$$
\bar{P}=q \cdot n_{q}+e_{q} \quad \text { and } \quad P=r \cdot n_{r}+e_{r}
$$

where $n_{q}, e_{q}, n_{r}, e_{r} \in \mathbb{N}$, and $e_{q}<q, e_{r}<r$. Equation (36) obviously corresponds to $e_{q}=e_{r}=0$, i.e., when $r$ divides $P$ and $q$ divides $\bar{P}$. For general values of $r$ and $q$, the block pseudo-circulant $\hat{\mathbf{E}}_{m}(z)$ from (36) gets transformed by inserting $e_{r}$ additional columns of zeros in each block-row and by adding $e_{q}$ additional rows at the bottom. In the following, we will assume $e_{q}=e_{r}=0$ since this leads to essentially no loss of generality. Furthermore, we will assume that $n_{q}=n_{r}$ or, equivalently, that $\bar{P}=q n_{r}$, which is a valid assumption since $\bar{P}$ is a free parameter.

\section{B. Eliminating IBI}

Next, we would like to eliminate the memory dependence in (36), which is responsible for inter-block interference (IBI). It is apparent from Fig. 9 that this can be achieved by choosing $\hat{\mathbf{C}}_{m}$ such that its last $r N$ rows are zero. This effectively means that the transmitter is inserting a redundancy of $r N$ symbols after each block of length $P-r N$. Let us denote by $\overline{\mathbf{E}}_{m}$ the $\bar{P} \times$ $(P-N r)$ constant matrix obtained as a result of premultiplying $\hat{\mathbf{C}}_{m}$ by $\hat{\mathbf{E}}_{m}(z)$. Next, we note that the blocked version of the equality (6) holds true as well. In other words, $\overline{\mathbf{E}}_{m}$ can be blockdiagonalized using block-Vandermonde matrices. Namely, let us choose

$$
\mathbf{G}_{m}=\left[\begin{array}{cccc}
\mathbf{I}_{q} & \rho_{m, 0}^{-1} \mathbf{I}_{q} & \cdots & \rho_{m, 0}^{-n_{q}+1} \mathbf{I}_{q} \\
\mathbf{I}_{q} & \rho_{m, 1}^{-1} \mathbf{I}_{q} & \cdots & \rho_{m, 1}^{-n_{q}+1} \mathbf{I}_{q} \\
\vdots & \vdots & & \vdots \\
\mathbf{I}_{q} & \rho_{m, J-1}^{-1} \mathbf{I}_{q} & \cdots & \rho_{m, J-1}^{-n_{q}+1} \mathbf{I}_{q}
\end{array}\right], \text { for } \rho_{m, j} \in \mathbb{C}
$$

denote by $\boldsymbol{\Theta}_{m}$ the following $J r \times(P-N r)$ matrix, recalling that $n_{r}=n_{q}$

$$
\boldsymbol{\Theta}_{m}=\left[\begin{array}{cccc}
\mathbf{I}_{r} & \rho_{m, 0}^{-1} \mathbf{I}_{r} & \cdots & \rho_{m, 0}^{-\left(n_{r}-N-1\right)} \mathbf{I}_{r} \\
\mathbf{I}_{r} & \rho_{m, 1}^{-1} \mathbf{I}_{r} & \cdots & \rho_{m, 1}^{-\left(n_{r}-N-1\right)} \mathbf{I}_{r} \\
\vdots & \vdots & & \vdots \\
\mathbf{I}_{r} & \rho_{m, J-1}^{-1} \mathbf{I}_{r} & \cdots & \rho_{m, J-1}^{-\left(n_{r}-N-1\right)} \mathbf{I}_{r}
\end{array}\right]
$$

and define the $q J \times r J$ block-diagonal matrix

$$
\mathcal{E}_{m}\left(\boldsymbol{\rho}_{m}\right) \triangleq \operatorname{diag}\left[\mathbf{E}_{m}\left(\rho_{m, 0}\right), \mathbf{E}_{m}\left(\rho_{m, 1}\right), \cdots, \mathbf{E}_{m}\left(\rho_{m, J-1}\right)\right] .
$$

Then, for any $J \in \mathbb{N}$ and any set of distinct complex numbers $\left\{\rho_{m, j}\right\}_{j=0}^{J-1}$, the following holds:

$$
\mathbf{G}_{m} \overline{\mathbf{E}}_{m}=\mathcal{E}_{m}\left(\boldsymbol{\rho}_{m}\right) \boldsymbol{\Theta}_{m} .
$$

Notice that the symbols $\mathbf{G}_{m}$ and $\Theta_{m}$ are used here to represent different matrices from the ones in Section II. This is done for notational simplicity since no confusion is anticipated.

Once we have established the connection with the traditional AMOUR systems, we follow the steps similar to those in
Section II in order to get conditions for MUI cancellation and channel equalization, regardless of the channels $h_{m}(n)$. Given the analogy between (41) and (6), we conjecture that the block at the receiver in Fig. 9 that is responsible for MUI elimination should be given by $\mathbf{G}_{m}$, as in (38). In the following, we first clarify this point and then proceed to state the result on the existence of channel ZFEs.

\section{MUI Cancellation}

The interference at the $m$ th receiver coming from the user $\mu \neq m$ is proportional to the output of the concatenation of matrices $\mathbf{G}_{m} \overline{\mathbf{E}}_{\mu} \mathbf{C}_{\mu}$, where $\mathbf{C}_{\mu}$ is the nonzero part of the spreading code matrix $\hat{\mathbf{C}}_{\mu}$ and is exactly the same as the one used in (7). Using (41), we see that the MUI term is proportional to

$$
\begin{aligned}
& \mathbf{G}_{m} \overline{\mathbf{E}}_{\mu} \mathbf{C}_{\mu} \\
& \quad=\mathcal{E}_{\mu}\left(\boldsymbol{\rho}_{m}\right) \boldsymbol{\Theta}_{m} \mathbf{C}_{\mu}=\mathcal{E}_{\mu}\left(\boldsymbol{\rho}_{m}\right) \mathcal{C}_{\mu}\left(\boldsymbol{\rho}_{m}\right) \quad \text { with } \\
& \mathcal{C}_{\mu}\left(\boldsymbol{\rho}_{m}\right) \\
& \quad \triangleq\left[\begin{array}{c}
\mathbf{C}_{\mu}\left(\rho_{m, 0}\right) \\
\mathbf{C}_{\mu}\left(\rho_{m, 1}\right) \\
\vdots \\
\mathbf{C}_{\mu}\left(\rho_{m, J-1}\right)
\end{array}\right], \quad \text { and } \\
& \quad \triangleq \quad\left[\begin{array}{cccc}
C_{\mu, 0}^{(0)}(\gamma) & C_{\mu, 1}^{(0)}(\gamma) & \cdots & C_{\mu, K-1}^{(0)}(\gamma) \\
C_{\mu, 0}^{(1)}(\gamma) & C_{\mu, 1}^{(1)}(\gamma) & \cdots & C_{\mu, K-1}^{(1)}(\gamma) \\
\vdots & \vdots & & \vdots \\
C_{\mu, 0}^{(r-1)}(\gamma) & C_{\mu, 1}^{(r-1)}(\gamma) & \cdots & C_{\mu, K-1}^{(r-1)}(\gamma)
\end{array}\right] .
\end{aligned}
$$

The entries $C_{\mu, k}^{(l)}(\gamma)$, for $0 \leq k \leq K-1,0 \leq l \leq r-1$ in (43), represent the $l$ th Type-1 polyphase components of the $k$ th spreading code used by user $\mu$, evaluated at $z=\gamma$. In other words, the $k$ th spreading code in Fig. 1(a) can be written as

$$
C_{m, k}(z)=\sum_{l=0}^{r-1} C_{m, k}^{(l)}\left(z^{r}\right) z^{-l} .
$$

It follows from (42) and (43) that MUI elimination can be achieved by choosing $\left\{\rho_{m, j}\right\}_{m, j=0}^{M-1, J-1}$ such that

$$
\begin{aligned}
& C_{\mu, k}^{(l)}\left(\rho_{m, j}\right)=0, \quad \forall m \neq \mu, \quad \forall k \in[0, K-1], \\
& \quad \forall j \in[0, J-1], \quad \forall l \in[0, r-1] .
\end{aligned}
$$

Equations (44) define $(M-1) J$ zeros for each of the $r$ polyphase components of $C_{m, k}(z)$. In addition to this, we will choose the nonzero values similarly as in Section II such that the channel equalization becomes easier. To this end, let us choose

$$
C_{m, k}^{(l)}\left(\rho_{m, j}\right)=A_{m} \cdot \delta(l-\beta) \cdot \rho_{m, j}^{-\alpha}
$$

for integers $\alpha$ and $\beta$ with $\beta<r$ chosen such that $k=\alpha r+$ $\beta$. This brings the total number of constraints in each of the spreading code polynomials to $M J r$. Recalling that the last $N r$ samples of spreading codes are fixed to be zero, the minimum spreading code length is given by $P=(M J+N) r$. 


\section{Channel Equalization}

The last step in the receiver design is to eliminate the ISI present in the MUI-free signal. For an arbitrary choice of integers $K$ and $r$ with $r<K$, we can write

$$
K=r \cdot \alpha_{r}+\beta_{r}
$$

with $\alpha_{r}, \beta_{r} \in \mathbb{N}$, and $\beta_{r}<r$. Let us first assume that $K$ was chosen such that $\beta_{r}=0$ in (46). Substituting (45) in (43) for $\mu=m$, we have

$$
\mathbf{C}_{m}\left(\rho_{m, j}\right)=A_{m}\left[\mathbf{I}_{r} \rho_{m, j}^{-1} \mathbf{I}_{r} \cdots \rho_{m, j}^{-\left(\alpha_{r}-1\right)} \mathbf{I}_{r}\right]
$$

which further leads to

$$
\begin{aligned}
\mathbf{G}_{m} \overline{\mathbf{E}}_{\mu} \mathbf{C}_{\mu}= & A_{m} \cdot \mathcal{E}_{\mu}\left(\boldsymbol{\rho}_{m}\right) \\
& \cdot\left[\begin{array}{cccc}
\mathbf{I}_{r} & \rho_{m, 0}^{-1} \mathbf{I}_{r} & \cdots & \rho_{m, 0}^{-\left(\alpha_{r}-1\right)} \mathbf{I}_{r} \\
\mathbf{I}_{r} & \rho_{m, 1}^{-1} \mathbf{I}_{r} & \cdots & \rho_{m, 1}^{-\left(\alpha_{r}-1\right)} \mathbf{I}_{r} \\
\vdots & \vdots & & \vdots \\
\mathbf{I}_{r} & \rho_{m, J-1}^{-1} \mathbf{I}_{r} & \cdots & \rho_{m, J-1}^{-\left(\alpha_{r}-1\right)} \mathbf{I}_{r}
\end{array}\right] .
\end{aligned}
$$

Recalling the relationship (41), we finally have that

$$
\begin{aligned}
& \mathbf{G}_{m} \overline{\mathbf{E}}_{m} \mathbf{C}_{m} \\
& =A_{m} \cdot \underbrace{\left[\begin{array}{cccc}
\mathbf{I}_{q} & \rho_{m, 0}^{-1} \mathbf{I}_{q} & \cdots & \rho_{m, 0}^{-\left(\alpha_{r}+N-1\right)} \mathbf{I}_{q} \\
\mathbf{I}_{q} & \rho_{m, 1}^{-1} \mathbf{I}_{q} & \cdots & \rho_{m, 1}^{-\left(\alpha_{r}+N-1\right)} \mathbf{I}_{q} \\
\vdots & \vdots & & \vdots \\
\mathbf{I}_{q} & \rho_{m, J-1}^{-1} \mathbf{I}_{q} & \cdots & \rho_{m, J-1}^{-\left(\alpha_{r}+N-1\right)} \mathbf{I}_{q}
\end{array}\right]}_{\mathbb{V}_{m}} \cdot \mathbb{E}_{m}
\end{aligned}
$$

where $\mathbb{E}_{m}$ is the $\left(\alpha_{r}+N\right) q \times K$ northwest submatrix of $\overline{\mathbf{E}}_{m}$. If $\beta_{r}>0$ in (46), this simply leads to adding the first $\beta_{r}$ columns of the next logical block to the right end in (47), consequently augmenting the matrices $\mathbb{V}_{m}$ and $\mathbb{E}_{m}$ in (49).

The channel equalization block, following the MUI cancellation, amounts to finding a left inverse of the matrix product $\mathbb{V}_{m} \cdot \mathbb{E}_{m}$ appearing on the right-hand side of (49). The first matrix in this product is block-Vandermonde, and it is invertible if $J \geq \alpha_{r}+N$ and if $\left\{\rho_{m, j}\right\}_{j=0}^{J-1}$ are distinct (the latter was assured previously). Therefore, we get the minimum value for one of the parameters

$$
J=\alpha_{r}+N \text {. }
$$

Notice that since $q>r$, from (50) and (46), it automatically follows that $\mathbb{V}_{m} \cdot \mathbb{E}_{m}$ is a tall matrix; thus, it could have a left inverse. However, these conditions are not sufficient. Another condition that needs to be satisfied is the following:

$$
\operatorname{rank}\left\{\mathbf{G}_{m} \overline{\mathbf{E}}_{m} \mathbf{C}_{m}\right\}=K \Rightarrow \operatorname{rank}\left\{\mathcal{E}_{m}\left(\boldsymbol{\rho}_{m}\right)\right\} \geq K
$$

In other words, in order for the channel $h_{m}(n)$ to be equalizable using ZFEs, the following needs to be satisfied. After oversampling the received signal by $q / r$ and MUI cancellation, we can allow for the rank of $\mathcal{E}_{m}\left(\boldsymbol{\rho}_{m}\right)$ in (40) to drop by the maximum amount of $r \cdot N$, regardless of the choice of signature points $\left\{\rho_{m, j}\right\}$. Obviously, this cannot be guaranteed regardless of the channel and other system parameters simply because the matrix polynomial $\mathbf{E}_{m}(z)$ could happen to be rank-deficient for all values of $z$. At best, we can only hope to establish the conditions under which the rank equality (51) stays satisfied, regardless of the choice of signature points. This is different from the conventional AMOUR and integral FSAMOUR methods described in Sections II and III, where we had two conditions on system parameters for guaranteed channel equalizability, depending on whether the channel was known $(J \geq K)$ or unknown $(J \geq K+L)$. Here, we cannot guarantee equalizability even for the known CSI, if the channel leads to rank-deficient $\mathbf{E}_{m}(z)$. Luckily, this occurs with zero probability. ${ }^{2}$ If $\mathbf{E}_{m}(z)$ is not rank-defficient, the channel can be equalized under the same restrictions on the parameters, regardless of the specific channel in question. The following theorem establishes the result under one extra assumption on the decimation ratio $r$.

Theorem 1: Consider the FSAMOUR communication system given by its discrete-time equivalent in Fig. 9(a). Let the maximum order of all the channels $\left\{h_{m}(n)\right\}_{m=0}^{M-1}$ be $L$. Let us choose the integers $r \geq L+1$ and $q>r$ such that the irreducible ratio $q / r$ closely approximates the desired amount of oversampling at the receiver. Next, choose an arbitrary $\alpha_{r} \geq r$, and take the following values of the parameters:

$K=r \cdot \alpha_{r}, \quad J=\alpha_{r}+1, \quad P=(M J+1) r, \quad \bar{P}=(M J+1) q$.

1) MUI can be eliminated by blocking the received signal into the blocks of length $\bar{P}$ and passing it through the matrix $\mathbf{G}_{m}$, as introduced in (38) with $n_{q}=M J+$ 1 , as long as the spreading codes $\left\{c_{m, k}(n)\right\}_{k=0}^{K-1}$ are chosen according to (44) and (45).

2) Under the above conditions, the channel can either be equalized for an arbitrary choice of the signature points $\left\{\rho_{m, j}\right\}$, or it cannot be equalized, regardless of this choice. More precisely, let $\mathbf{E}_{m}(z)$ be the polyphase matrix corresponding to $h_{m}(n)$, as derived in (32)-(35). Under the above conditions, there are two possible scenarios:

- $\max _{z \in \mathbb{C}} \operatorname{rank}\left\{\mathbf{E}_{m}(z)\right\}=r$. In this case, the system is ZFE-equalizable, regardless of $\left\{\rho_{m, j}\right\}$.

- $\max _{z \in \mathbb{C}} \operatorname{rank}\left\{\mathbf{E}_{m}(z)\right\}<r$. In this case, there is no choice of $\left\{\rho_{m, j}\right\}$ that can make the system ZFE-equalizable.

Comment: The condition $r \geq L+1$ introduced in the statement of the theorem might seem restrictive at first. However, in most cases, it is of special interest to minimize the amount of oversampling at the receiver and try to optimize the performance under those conditions. This amounts to keeping $q$ roughly equal to yet slightly larger than $r$ and choosing $r$ large enough so that the ratio $q / r$ approaches unity. In such cases, $r$ happens to be greater than $L+1$ by design. The condition $\alpha_{r} \geq r$ is not necessary for the existence of ZFEs. It only ensures the absence of ZFEs if the rank condition on $\mathbf{E}_{m}(z)$ is not satisfied.

${ }^{2}$ Moreover, unless $\mathbf{E}_{m}(z)$ is rank-deficient, even if it happens to be ill-conditioned for certain values of $\rho_{m, j}$, for known CSI, this can be avoided by the appropriate choice of signature points. 


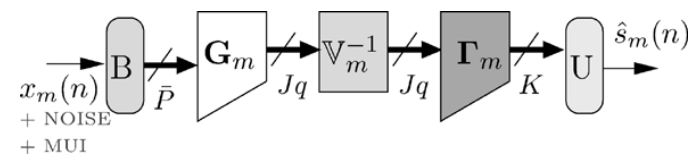

Fig. 10. Proposed structure of the FSAMOUR receiver in systems with fractional oversampling.

Proof: The only result that needs proof in the first part of the theorem is that the order of $\mathbf{E}_{m}(z)$ is $N=1$, whenever $r \geq L+1$. If $N=1$, all the parameters in (52) are consistent with the values used so far in Section IV. Then, the first claim follows directly from the discussion preceding the theorem. In order to prove that $N=1$, we use the following lemma, whose proof can be found in the Appendix.

Lemma 1: Under the conditions of Theorem $1, \mathbf{E}_{m}(z)$ can be written as

$$
\mathbf{E}_{m}(z)=\mathbf{U}_{m} \cdot \mathbf{D}_{m}(z) \cdot\left[\begin{array}{l}
\mathbf{E}_{m, 0}^{r}(z) \\
\mathbf{E}_{m, 1}(z)
\end{array}\right] \begin{aligned}
& r \\
& q-r
\end{aligned}
$$

where $\mathbf{E}_{m, 0}(z)$ and $\mathbf{E}_{m, 1}(z)$ are polynomial matrices of order $N=1, \mathbf{U}_{m}$ is a unitary matrix, and $\mathbf{D}_{m}(z)$ is a diagonal matrix with advance operators $z^{i}$ on the diagonals.

Having established Lemma 1, the first part of the theorem follows readily since $\mathbf{U}_{m} \cdot \mathbf{D}_{m}(z)$ can be equalized effortlessly, and thus, the order of $\mathbf{E}_{m}(z)$ is indeed $N=1$ for all practical purposes.

For the second part of Theorem 1, we use Lemma 2, which is also proved in the Appendix.

Lemma 2: The difference between the maximum and the minimum achievable rank of $\mathcal{E}_{m}\left(\rho_{m}\right)$ given by (40) is upper bounded by $r-1$.

From the proof of Lemma 2, it follows that we can distinguish between two cases.

- If the normal rank of $\mathbf{E}_{m}(z)$ is $r$, then the minimum rank of $\mathcal{E}_{m}\left(\boldsymbol{\rho}_{m}\right)$ over all choices of signature points is lower bounded by $r J-r+1=K+1$, and therefore, ZFE is achieved by finding a left inverse of the product in (49).

- If the normal rank of $\mathbf{E}_{m}(z)$ is less than $r$, then the maximum rank of $\mathcal{E}_{m}\left(\boldsymbol{\rho}_{m}\right)$ is given by

$$
\begin{aligned}
\max _{\rho_{m}, j} \operatorname{rank}\left\{\mathcal{E}_{m}\left(\boldsymbol{\rho}_{m}\right)\right\} & \leq(r-1) J \\
& =(r-1)\left(\alpha_{r}+1\right) \\
& =K+\left(r-\alpha_{r}-1\right)<K .
\end{aligned}
$$

Therefore, regardless of the signature points, ZFE does not exist.

This concludes the proof of Theorem 1.

$\nabla \nabla \nabla$

To summarize, in this section, we established the algorithm for multiuser communications based on AMOUR systems with fractional amount of oversampling at the receiver. The proposed form of the receiver (block labeled "equalization and rate reduction" in Fig. 9) is shown in Fig. 10. As was the case with the simple AMOUR systems, the receiver is divided into three parts, namely $\mathbf{G}_{m}, \mathbb{V}_{m}^{-1}$, and $\boldsymbol{\Gamma}_{m}$. The first block $\mathbf{G}_{m}$ is supposed to eliminate MUI at the receiver. The second block $\mathbb{V}_{m}^{-1}$ represents the inverse of $\mathbb{V}_{m}$, which is defined in (49) and essentially neutralizes the effect of $\hat{\mathbf{C}}_{m}$ and $\mathbf{G}_{m}$ on the MUI-free signal. Finally, $\boldsymbol{\Gamma}_{m}$ is the block designed to equalize the channel that is now embodied in the tall matrix $\mathbb{V}_{m}$ [see (49)].

Note that even though the notations may be similar as in Section II, the building blocks in Fig. 10 are quite different from the corresponding ones in AMOUR systems. The construction of $\mathbf{G}_{m}$ is described in (38) with the signature points chosen in accordance with the spreading code constraints (44) and (45). The channel equalizer $\boldsymbol{\Gamma}_{m}$ can be chosen according to one of the several design criteria described in (17). Instead of $\overline{\mathbf{H}}_{m}$ in (17), we should use the corresponding matrix $\mathbb{E}_{m}$. In addition to these three conventional solutions, we can choose the optimal zero-forcing equalizer as the one described in Section III-A. The details of the construction of this solution are omitted since they are analogous to the derivations in Section III-A.

The conditions for the existence of any ZFE $\boldsymbol{\Gamma}_{m}^{(\mathrm{zfe})}$ are described Theorem 1. Under the same conditions, there will exist the optimal ZFE $\Gamma_{m}^{(\mathrm{opt})}$ as well. The event that the normal rank of $\mathbf{E}_{m}(z)$ is less than $r$ occurs with zero probability, and thus, for all practical purposes, we can assume that the channel is equalizable, regardless of the choice of signature points. Again, for the reasons of computational benefits, signature points can be chosen to be uniformly distributed on the unit circle [see (10)]. In the following, we demonstrate the advantages of the FSAMOUR systems with fractional oversampling over the conventional AMOUR systems.

\section{E. Performance Evaluation}

In this section, we present the simulation results comparing the performance of the conventional AMOUR system to the FSAMOUR system with a fractional oversampling ratio. The simulation resuts are averaged over 30 independently chosen real random channels of order $L=4$. The $q$-times oversampled channel impulse responses $h_{m}^{(q)}(n)$ were also chosen randomly under the constraint that they coinside with AMOUR channels at integers. In other words, $h_{m}^{(q)}(q n)=h_{m}(n)$. The channel noise was taken to be colored. However, as opposed to Section III-B, it was modeled as an auto-regressive process of first order [11], i.e., AR(1) process with the cross-correlation coefficient equal to 0.8 . The SNR was measured at the receiver, as explained in Section III-B. The amount of oversampling at the receiver was chosen to be $q / r=6 / 5$ and the parameter $\alpha_{r}=6$. The other parameters were chosen as in (52). Notice that the advantage of this system over the one described in Section III is in the lower data rate at the receiver. Namely, for each five symbols of the input data stream $s_{m}(n)$, the receiver in Fig. 3 needs to deal with ten symbols, whereas the receiver in Fig. 9 deals with only six. This represents not only the reduction in complexity of the receiver but also minimizes the additional on-chip RF noise resulting from fast-operating integrated circuits.

The performance curves are shown in Fig. 11. The acronyms "SSE" and "FSE" represent the AMOUR system with no oversampling and the FSAMOUR system with the oversampling ratio 6/5, whereas the suffixes "ZF," "MMSE," and "OPT" 


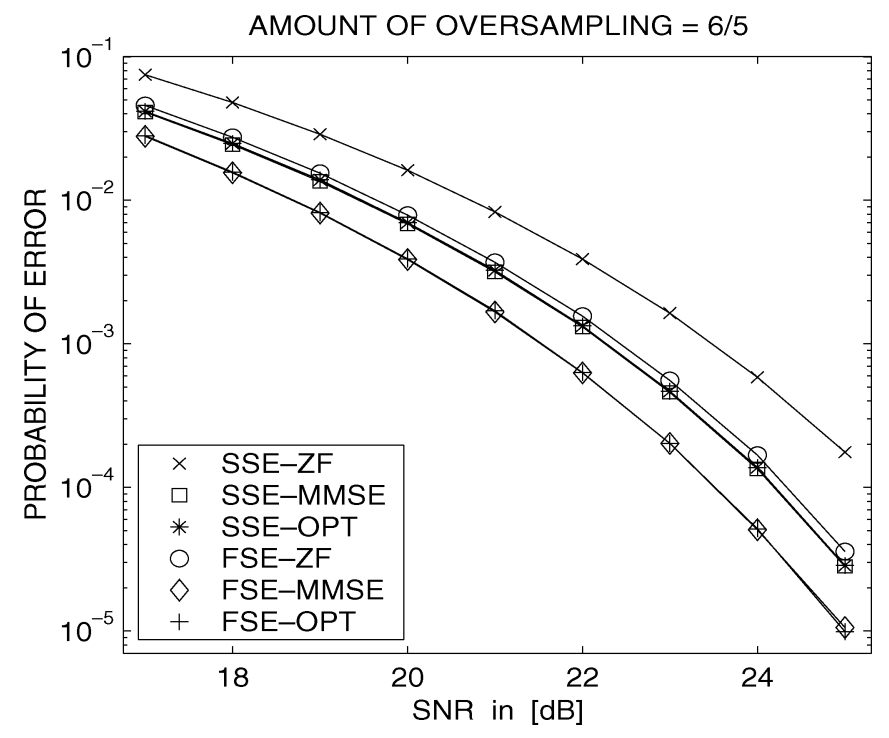

Fig. 11. Probability of error as a function of SNR in AMOUR and FSAMOUR systems with oversampling ratio $6 / 5$.

correspond to the zero-forcing, minimum mean-squared error, and optimal ZFE solutions, respectively. The optimal ZFEs are based on optimal matrix inverses, as explained in Section III-A. Comparing these performances, we conclude the following.

- In this case (due to noise coloring and fractional oversampling), the optimal ZFE in both the AMOUR and FSAMOUR systems perform significantly better than the conventional ZFE. This comes in contrast to some of the results in Section III-B.

- $\quad$ The optimal ZFEs in both systems on Fig. 11 perform almost identically to the MMSE solutions. As explained in Section III-B, the complexity of $\Gamma_{m}^{(\mathrm{opt})}$ is reduced compared with that of $\Gamma_{m}^{(\mathrm{mmse})}$ and so is the required knowledge of the signal and the noise statistics.

- $\quad$ The FSAMOUR system with the oversampling ratio 6/5 performs better than the corresponding AMOUR system with no oversampling. The price to be paid is in the data rate and the complexity at the receiver. As expected, the improvement in performance resulting from oversampling by a ratio $6 / 5$ is not as pronounced as in Section III-B, with a ratio $q=2$. This can be assessed by comparing the gain over the symbol-spaced system in Figs. 7 and 11).

\section{CONCluding Remarks}

The recent development of A Mutually Orthogonal Usercode Receiver (AMOUR) for asynchronous or quasisynchronous CDMA systems [3], [4] represents a major break-through in the theory of multiuser communications. The main advantage over some of the other methods lies in the fact that both the suppression of MUI and ISI within a single user can be achieved, regardless of the multipath channels. For this reason, it is very easy to extend the AMOUR method to the case where these channels are unknown [4]. In this paper, we proposed a modification of the traditional AMOUR system in that the received continuous-time signal is oversampled by an integral or a rational amount. This idea leads to the concept of Fractionally Spaced AMOUR (FSAMOUR) receivers that are derived for both integral and rational amounts of oversampling. Their performance is compared to the corresponding performance of the conventional method, and significant improvements are observed. An important point often overlooked in the design of zero-forcing channel equalizers is that sometimes, they are not unique. We exploit this flexibility in the design of AMOUR and FSAMOUR receivers and further improve the performance of multiuser communication systems.

\section{APPENDIX}

Proof of Lemma 1: Without loss of generality, we only consider $r=L+1$ since the proof for $r>L+1$ follows essentially the same lines. The polyphase components $H_{m, k}(z)$ of the $q$-fold oversampled channel $H^{(q)}(z)$ defined in (32) can be thought of as FIR filters of order $L$ (or less). As a special case, note that $H_{m, 0}(z)=H_{m}(z)$. Next, consider the auxiliary filters $P_{m, k}(z)$, as in (34). From (33), it follows not only that $q$ and $r$ are coprime but, at the same time, that $Q$ and $r$ are coprime as well. For this reason, the numbers

$$
l_{k} \triangleq[k Q \bmod \quad r]
$$

are distinct for each $0 \leq k \leq r-1$. As a consequence, the first $r$ filters

$$
P_{m, k}(z)=z^{k Q} H_{m, k}(z), \quad 0 \leq k \leq r-1
$$

of length $L+1$ are delayed by the amounts that are all different relative to the start of blocks of length $r$. This, combined with the fact that $r=L+1$, leads us to conclude that the entries of $\mathbf{E}_{m}(z)$, namely $E_{k, l}(z)$ defined in (35), are all given by

$$
E_{k, l}(z)=\tilde{e}_{k, l} \cdot z^{n_{k, l}} .
$$

Here, $\tilde{e}_{k, l}$ are constants, $n_{k, l} \geq 0, n_{k, l+1} \geq n_{k, l}$, and $n_{k, r-1} \leq$ $n_{k, 0}+1$. Moreover, the index within the $k$ th row of $\mathbf{E}_{m}(z)$, where the exponent $n_{k, l}$ increases by one, is different for each of the first $r$ rows, and all the polyphase components $E_{k, l}(z)$ for $k=0$ are constant. It follows that indeed, $\mathbf{E}_{m}(z)$ can be written as (53), with $\mathbf{U}_{m}$ denoting the unitary matrix corresponding to row permutations and $\mathbf{D}_{m}(z)$ given by

$$
\mathbf{D}_{m}(z)=\operatorname{diag}\left[\begin{array}{llll}
z^{m_{0}} & z^{m_{1}} & \cdots & z^{m_{q-1}}
\end{array}\right], \quad m_{k} \in \mathbb{N}
$$

whose purpose is to pull out any common delay elements from each row of $\mathbf{E}_{m}(z)$.

$\nabla \nabla \nabla$

Proof of Lemma 2: Consider (53). Depending on $\mathbf{U}_{m}$, $\mathbf{E}_{m, 0}(z)$ can be chosen as

$$
\mathbf{E}_{m, 0}(z)=\left[\begin{array}{ccccc}
e_{0,0} & e_{0,1} & e_{0,2} & \cdots & e_{0, r-1} \\
e_{1,0} & z \cdot e_{1,1} & z \cdot e_{1,2} & \cdots & z \cdot e_{1, r-1} \\
e_{2,0} & e_{2,1} & z \cdot e_{2,2} & \cdots & z \cdot e_{2, r-1} \\
\vdots & \vdots & \vdots & \ddots & \vdots \\
e_{r-1,0} & e_{r-1,1} & e_{r-1,2} & \cdots & z \cdot e_{r-1, r-1}
\end{array}\right] .
$$

From (55), it follows that

$$
\operatorname{ord}\left\{\operatorname{det}\left[\mathbf{E}_{m, 0}(z)\right]\right\} \leq r-1 \text {. }
$$


Therefore, (55) can be rewritten using the Smith-McMillan form for the FIR case [14]

$$
\mathbf{E}_{m, 0}(z)=\hat{\mathbf{U}}_{0}(z) \Lambda_{0}(z) \hat{\mathbf{V}}_{0}(z)
$$

where $\hat{\mathbf{U}}_{0}(z)$ and $\hat{\mathbf{V}}_{0}(z)$ are unimodular, and $\boldsymbol{\Lambda}_{0}(z)$ is diagonal with polynomials $\lambda_{i}(z)$ on the diagonal for $0 \leq i \leq r-1$. From (56), it follows that

$$
\sum_{i=0}^{r-1} \operatorname{ord}\left\{\lambda_{i}(z)\right\} \leq r-1 .
$$

Note that some of the diagonal polynomials $\lambda_{i}(z)$ can be identically equal to zero, which will result in $\operatorname{rank}\left\{\mathbf{E}_{m, 0}(\gamma)\right\}<r$, regardless of $\gamma$. However, if this is not the case, it follows from (58) that by varying $z$, the rank of $\mathbf{E}_{m, 0}(z)$ can drop by at most $r-1$. This concludes the proof.

$\nabla \nabla \nabla$

\section{REFERENCES}

[1] I. Ghauri and D. T. M. Slock, "Blind maximum SINR receiver for the DS-CDMA downlink," in Proc. ICASSP, Istanbul, Turkey, Jun. 2000.

[2] G. B. Giannakis, Y. Hua, P. Stoica, and L. Tong, Eds., Signal Processing Advances in Wireless and Mobile Communications-Volume I, Trends in Channel Estimation and Equalization. Englewood Cliffs, NJ: Prentice-Hall, Sep. 2000.

[3] G. B. Giannakis, Z. Wang, A. Scaglione, and S. Barbarossa, "AMOUR - generalized multicarrier CDMA irrespective of multipath," in Proc. Globecom, Rio de Janeiro, Brazil, Dec. 1999.

[4] G. B. Giannakis, Z. Wang, A. Scaglione, and S. Barbarossa, "AMOUR - generalized multi-carrier transceivers for blind CDMA regardless of multipath," IEEE Trans. Commun., vol. 48, no. 12, pp. 2064-2076, Dec. 2000.

[5] R. A. Horn and C. R. Johnson, Matrix Analysis. New York: Cambridge Univ. Press, 1985.

[6] T. Kailath, Linear Systems. Englewood Cliffs, NJ: Prentice Hall, 1980.

[7] A. Klein, G. K. Kaleh, and P. W. Baier, "Zero forcing and minimum mean square error equalization for multiuser detection in code division multiple access channels," IEEE Trans. Veh. Technol., vol. 45, no. 2, pp. 276-287, May 1996.

[8] E. Moulines, P. Duhamel, J. Cardoso, and S. Mayrargue, "Subspace methods for the blind identification of multichannel FIR filters," IEEE Trans. Signal Process., vol. 43, no. 2, pp. 516-525, Feb. 1995.

[9] A. Scaglione and G. B. Giannakis, "Design of user codes in QS-CDMA systems for MUI elimination in unknown multipath," IEEE Commun. Lett., vol. 3, no. 2, pp. 25-27, Feb. 1999.

[10] A. Scaglione, G. B. Giannakis, and S. Barbarossa, "Redundant filterbank precoders and equalizers part II: blind channel estimation, synchronization and direct equalization," IEEE Trans. Signal Process., vol. 47, no. 7, pp. 2007-2022, Jul. 1999.

[11] C. W. Therrien, Discrete Random Signals and Statistical Signal Processing. Englewood Cliffs, NJ: Prentice Hall, 1992.

[12] J. R. Treichler, I. Fijalkow, and C. R. Johnson Jr., "Fractionally spaced equalizers: how long should they really be?," IEEE Signal Process. Mag., vol. 13, no. 3, pp. 65-81, May 1996.

[13] M. K. Tsatsanis, "Inverse filtering criteria for CDMA systems," IEEE Trans. Signal Process., vol. 45, no. 1, pp. 102-112, Jan. 1997.

[14] P. P. Vaidyanathan, Multirate Systems and Filter Banks. Englewood Cliffs, NJ: Prentice-Hall, 1995.

[15] P. P. Vaidyanathan and B. Vrcelj, "Theory of fractionally spaced cyclicprefix equalizers," in Proc. ICASSP, Orlando, FL, May 2002.

[16] S. Verdú, Multiuser Detection. Cambridge, U.K.: Cambridge Univ. Press, 1998

[17] B. Vrcelj and P. P. Vaidyanathan, "MIMO biorthogonal partners and applications," IEEE Trans. Signal Processing, vol. 50, no. 3, pp. 528-543, Mar. 2002.

[18] — - "Fractional biorthogonal partners and application to signal interpolation," in Proc. ISCAS, Scottsdale, AZ, May 2002.

[19] _ " "Fractional biorthogonal partners in fractionally spaced equalizers," in Proc. ICASSP, Orlando, FL, May 2002.

[20] - "Fractional biorthogonal partners in channel equalization and signal interpolation," IEEE Trans. Signal Process., vol. 51, no. 7, pp. 1928-1940, Jul. 2003.
[21] — , "On the general form of FIR MIMO biorthogonal partners," in Proc. 35th Asilomar Conf., Pacific Grove, CA, Nov. 2001.

[22] Z. Wang and G. B. Giannakis, "Block precoding for MUI/ISI-resilient generalized multicarrier CDMA with multirate capabilities," IEEE Trans. Commun., vol. 49, no. 11, pp. 2016-2027, Nov. 2001.

[23] S. Zhou, G. B. Giannakis, and C. Le Martret, "Chip-interleaved blockspread code division multiple access," IEEE Trans. Commun., vol. 50, no. 2, pp. 235-248, Feb. 2002.



Bojan Vrcelj (S'99-M'04) was born in Belgrade, Yugoslavia, in 1974. He received the B.S. degree in electrical engineering from the University of Belgrade in 1998 and the M.S. and Ph.D. degrees in electrical engineering from California Institute of Technology, Pasadena, in 1999 and 2003, respectively.

Since August 2003, he has been with Qualcomm Inc., San Diego, CA. His research interests include multirate signal processing and applications in digital communications, especially channel equalization, multicarrier and multiuser communication systems, as well as wavelets, signal interpolation, and sampling theory.

Dr. Vrcelj received the Graduate Division Fellowship in 1998 and the Schlumberger Fellowship in 2002, both at the California Institute of Technology.

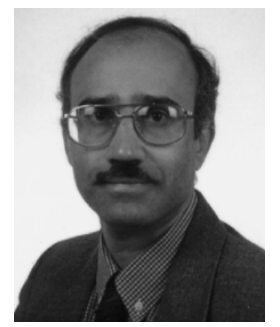

P. P. Vaidyanathan (S'80-M'83-SM'88-F'91) was born in Calcutta, India, on October 16, 1954. He received the B.Sc. (Hons.) degree in physics and the B.Tech. and M.Tech. degrees in radiophysics and electronics, all from the University of Calcutta, in 1974, 1977, and 1979, respectively, and the Ph.D. degree in electrical and computer engineering from the University of California, Santa Barbara, in 1982.

He was a post doctoral fellow at the University of California, Santa Barbara, from September 1982 to March 1983. In March 1983, he joined the Electrical Engineering Department, Calfornia Institute of Technology (Caltech), as an Assistant Professor, where since 1993, he has been Professor of electrical engineering. His main research interests are in digital signal processing, multirate systems, wavelet transforms, and signal processing for digital communications. $\mathrm{He}$ is a consulting editor for the journal Applied and Computational Harmonic Analysis.

Dr. Vaidyanathan served as Vice-Chairman of the Technical Program committee for the 1983 IEEE International Symposium on Circuits and Systems and as the Technical Program Chairman for the 1992 IEEE International Symposium on Circuits and Systems. He was an Associate Editor for the IEEE TRANSACTIONS ON CIRCUITS AND SYSTEMS from 1985 to 1987 and is currently an associate editor for the IEEE SIGNAL PROCESSING LETTERS. He was a guest editor in 1998 for special issues of the IEEE TRANSACTIONS ON SIGNAL PROCESSING and the IEEE TRANSACTIONS ON CIRCUITS AND SYSTEMS II on the topics of filterbanks, wavelets, and subband coders. He has authored a number of papers in IEEE journals and is the author of the book Multirate Systems and Filter Banks (Englewood Cliffs, NJ: Prentice-Hall, 1993). He has written several chapters for various signal processing handbooks. He was a recepient of the Award for Excellence in Teaching from the California Institute of Technology for the years 1983-1984, 1992-1993, and 1993-1994. He also received the NSF's Presidential Young Investigator Award in 1986. In 1989, he received the IEEE ASSP Senior Award for his paper on multirate perfect-reconstruction filterbanks. In 1990, he was recepient of the S. K. Mitra Memorial Award from the Institute of Electronics and Telecommuncations Engineers, India, for his joint paper in the IETE Journal. He was also the coauthor of a paper on linear-phase perfect reconstruction filterbanks in the IEEE TRANSACTIONS ON SIGNAL PROCESSING, for which the first author (T. Nguyen) received the Young Outstanding Author Award in 1993. He received the 1995 F. E. Terman Award of the American Society for Engineering Education, sponsored by Hewlett Packard Co., for his contributions to engineering education, especially the book Multirate Systems and Filter Banks. He has given several plenary talks including the Sampta'01, Eusipco'98, SPCOM'95, and Asilomar'88 conferences on signal processing. He was chosen as a distinguished lecturer for the IEEE Signal Processing Society for the year 1996-1997. In 1999 , he received the IEEE CAS Society's Golden Jubilee Medal, and in 2002, he received the IEEE Signal Processing Society's Technical Achievement Award. 